مو مجلة التربية والعلم - المجلد (21) العدد (2) لسنة 2008 هـ

الحل الدوري لبعض أنظمة المعادلات التكاملية-التفاضلية اللاخطية من

\title{
نوع فولتيّا
}

رعد نوري بطرس وغادة شكر جميل

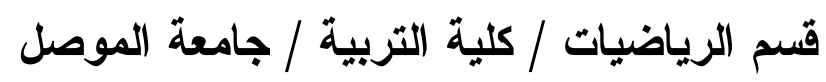

$$
\text { القبول }
$$

09/05/2007

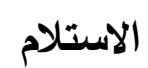

09/11/2006

\section{ABSTRACT}

In this paper we investigate the existence and approximation of the periodic solution for a system of nonlinear integro-differential equations of Volterra type. The numerical-analytic method has been used to study the periodic solutions of the non-linear ordinary differential equations that were introduced by A.M. Samoilenko.

\section{الملخص}

في هذا البحث تم در اسة وجود وتقريــب الحــل الــدوري لنظــام مــن المعــادلات

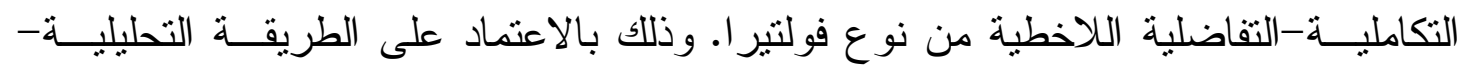

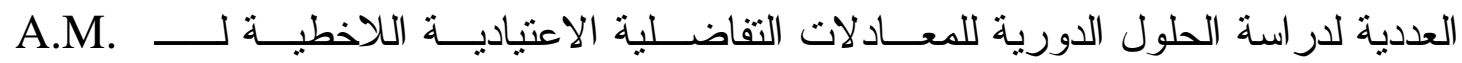

.Samoilenko

\section{المقدمة}

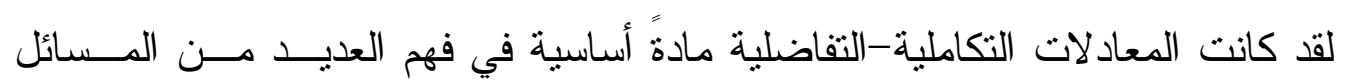

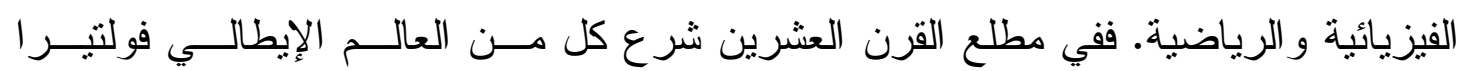
(V. Volterra)

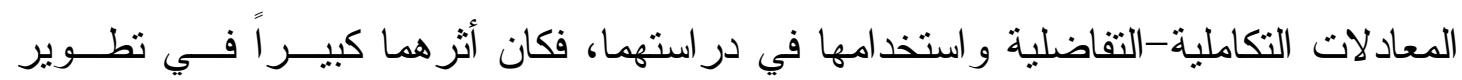
المعادلات التكاملية-التفاضلية الني كان لها دور بارز في بناء التحليل الرياضي و الدالي [3].

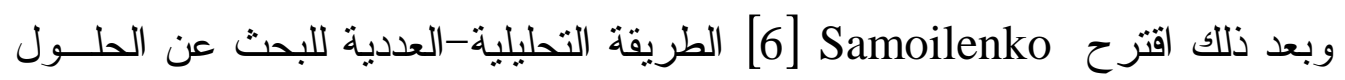

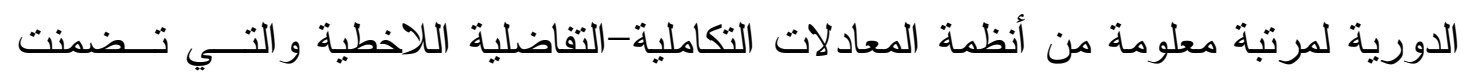

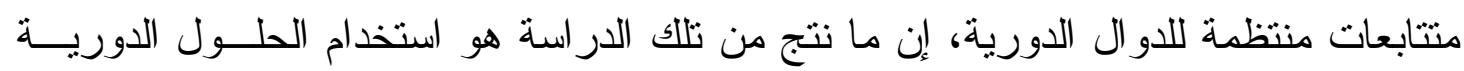
وبثكل واسع النطاق [2,1] في مختلف المعالجات العلمية و العطلية. 


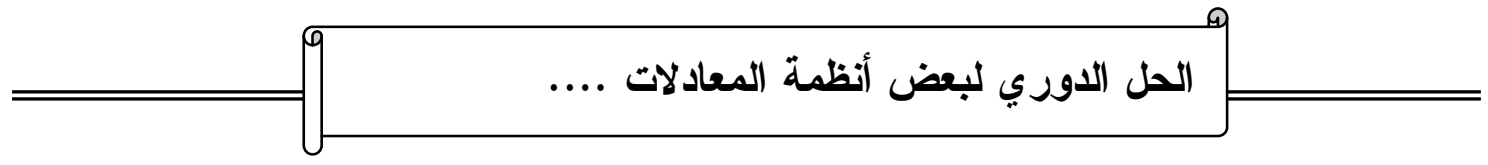

ندرس في هذا البحث نظام المعادلات التكامليــة-التفاضلية اللاخطية من الثكل أدناه: $\frac{d x}{d t}=A(t) x(t)+f\left(t, x, y, \int_{-\infty}^{t} u(t, s, \tau) h(\tau, x(\tau), y(\tau)) d \tau\right)$
$\frac{d y}{d t}=B(t) y(t)+g\left(t, x, y, \int_{-\infty}^{t} u(t, s, \tau) h(\tau, x(\tau), y(\tau)) d \tau\right)$

· $z \in D_{2} \subseteq R^{n}$ ، $y \in D_{1} \subseteq R^{n}$ ، $x \in D \subseteq R^{n}$ نيث ان

وان D ميثل مجالاً مغلقاً ومقيداً، و الدالتان المتجهنان (t, : $z=\int_{-\infty}^{t} u(t, s, \tau) h(\tau, x(\tau), y(\tau)) d \tau$ $(t, x, y, z) \in R^{1} \times D \times D_{1} \times D_{2}$

مستمرتان ودوريتان في t ذات دور يساوي T. إذ ان 1 ، في الفضـــاء الأقليدي بافتر اض أن كل الدوال في النظام (1) تحقق المتباينات الآتية: $\|f(t, x, y, z)\| \leq M_{1} \quad, \quad\|g(t, x, y, z)\| \leq M_{2}$

$\left\|f\left(t, x_{1}, y_{1}, z_{1}\right)-f\left(t, x_{2}, y_{2}, z_{2}\right)\right\| \leq K_{1}\left\|x_{1}-x_{2}\right\|+K_{2}\left\|y_{1}-y_{2}\right\|+K_{3}\left\|z_{1}-z_{2}\right\|$

$\left\|g\left(t, x_{1}, y_{1}, z_{1}\right)-g\left(t, x_{2}, y_{2}, z_{2}\right)\right\| \leq L_{1}\left\|x_{1}-x_{2}\right\|+L_{2}\left\|y_{1}-y_{2}\right\|+L_{3}\left\|z_{1}-z_{2}\right\|$

$\left\|h\left(\tau, x_{1}, y_{1}\right)-h\left(\tau, x_{2}, y_{2}\right)\right\| \leq N_{1}\left\|x_{1}-x_{2}\right\|+N_{2}\left\|y_{1}-y_{2}\right\|$

لكل كل مــن

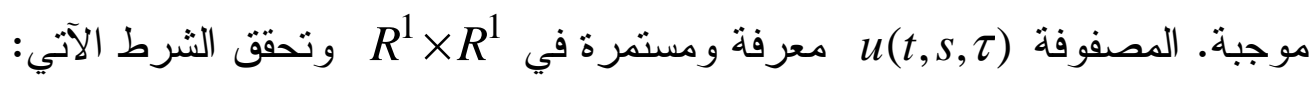
$u(t+T, s+T, \tau+T)=u(t, s, \tau)$

وبافتر اض ان كل من $n$ م

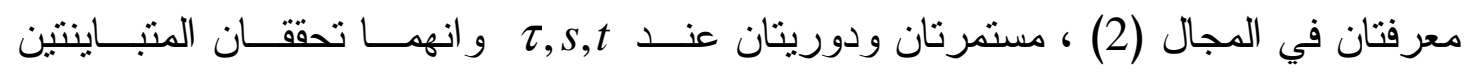

$\left\|e^{A(t-s-\tau)}\right\| \leq Q_{1} \quad, \quad\left\|e^{B(t-s-\tau)}\right\| \leq Q_{2}$

الآتيتين:

، حيث ان

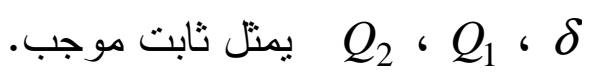
سوف نعرف المجاميع غير الخالية كالآتي: 


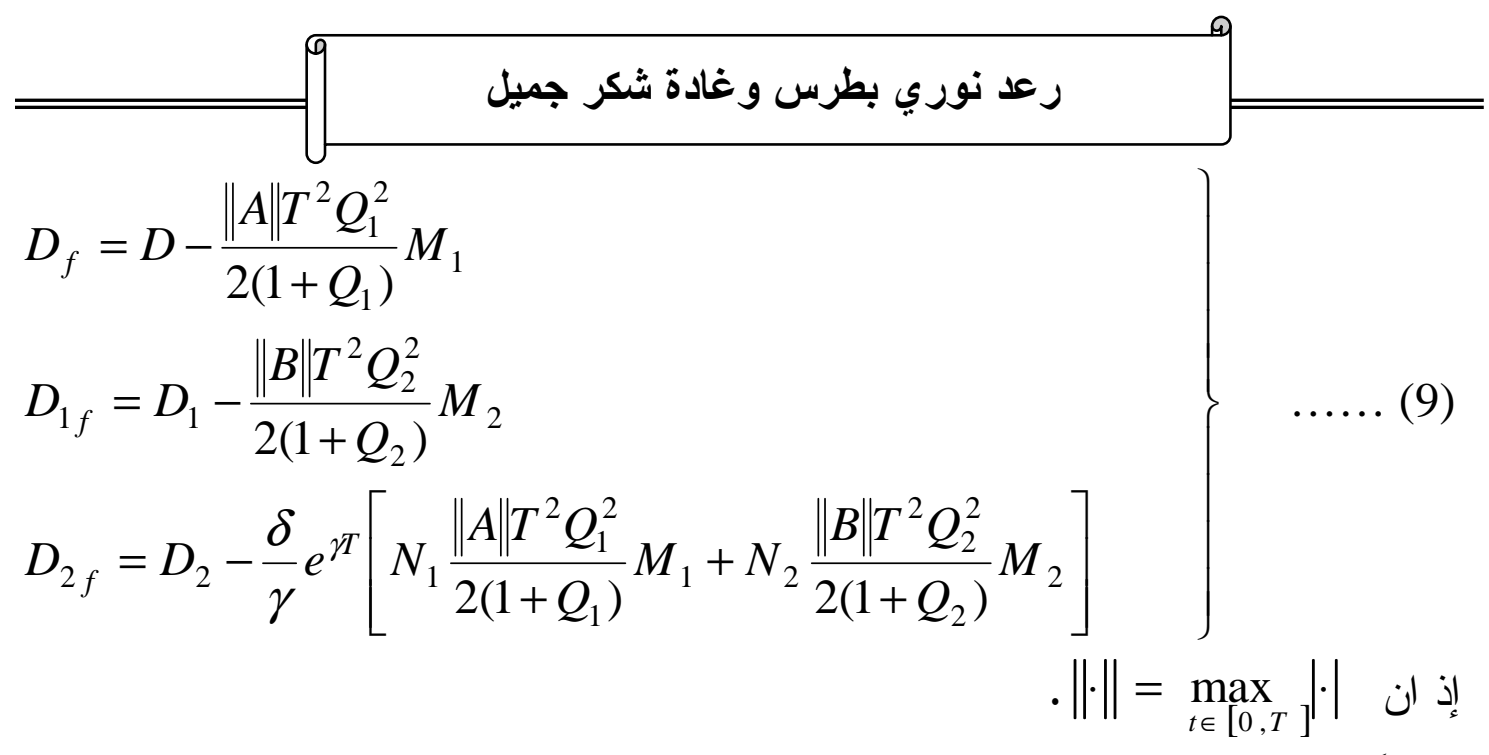

فضلاًً عن ذلك نفترض ان القيمة العظمى للمصفوفة الآتية: $\Lambda_{0}=\left(\begin{array}{ll}\frac{\|A\| T^{2} Q_{1}^{2}}{2\left(1+Q_{1}\right)}\left(K_{1}+K_{3} \frac{\delta}{\gamma} e^{\gamma T} N_{1}\right) & \frac{\|A\| T^{2} Q_{1}^{2}}{2\left(1+Q_{1}\right)}\left(K_{2}+K_{3} \frac{\delta}{\gamma} e^{\gamma T} N_{2}\right) \\ \frac{\|B\| T^{2} Q_{2}^{2}}{2\left(1+Q_{2}\right)}\left(L_{1}+L_{3} \frac{\delta}{\gamma} e^{\gamma T} N_{1}\right) & \frac{\|B\| T^{2} Q_{2}^{2}}{2\left(1+Q_{2}\right)}\left(L_{2}+L_{3} \frac{\delta}{\gamma} e^{\gamma T} N_{2}\right)\end{array}\right)$ أقل من الو احد أي ان $\lambda_{\max }\left(\Lambda_{0}\right)<1$

مأخوذة 1 :

لتكن ذات دور يساوي T عندئذ المنباينة الآتية: $\left\|\int_{0}^{t} e^{A(t-s-\tau)}\left[f(\tau, x, y, z)-\int_{0}^{T} \frac{A}{E+e^{A T}} e^{A(T-s-\tau)} f(\tau, x, y, z) d \tau\right] d \tau\right\| \leq \alpha_{1}(t) M_{1}$ $M_{1}=\max _{t}\|f(t, x, y, z)\| \quad$ تتحقق لكل $\alpha_{1}(t) \leq \frac{\|A\| T^{2} Q_{1}^{2}}{2\left(1+Q_{1}\right)}$ ، حيث ان $t \in[0, T]$ - $\alpha_{1}(t)=\left(t Q_{1}+\frac{\|A\|\left(T^{2}-2 t^{2}\right) Q_{1}^{2}}{2\left(1+Q_{1}\right)}\right)$,

من المتباينة الآتية:

$\left\|\int_{0}^{t} e^{A(t-s-\tau)}\left[f(\tau, x, y, z)-\int_{0}^{T} \frac{A}{E+e^{A T}} e^{A(T-s-\tau)} f(\tau, x, y, z) d \tau\right] d \tau\right\| \leq$ 


$$
\begin{aligned}
& \text { | } \\
& \leq \int_{0}^{t}\left\|e^{A(t-s-\tau)}\left(E-\frac{A \tau}{E+e^{A T}} e^{A(t-s-\tau)}\right) f(\tau, x, y, z)\right\| d \tau+\int_{t}^{T}\left\|\frac{A \tau}{E+e^{A T}} e^{2 A(t-s-\tau)} f(\tau, x, y, z)\right\| d \tau \\
& \text { نحصل على } \\
& \leq \int_{0}^{t}\left\|\left(E-\frac{A \tau}{E+e^{A T}} e^{A(t-s-\tau)}\right) e^{A(t-s-\tau)}\right\| f(\tau, x, y, z)\left\|d \tau+\int_{t}^{T}\right\| \frac{A \tau}{E+e^{A T}} e^{2 A(t-s-\tau)}\|\| f(\tau, x, y, z) \| d \tau \\
& \leq \int_{0}^{t}\left(1-\frac{\|A\| \tau}{1+Q_{1}} Q_{1}\right) Q_{1} M_{1} d \tau+\int_{t}^{T} \frac{\|A\| \tau}{1+Q_{1}} Q_{1}^{2} M_{1} d \tau \\
& =t Q_{1} M_{1}-\frac{\|A\| t^{2} Q_{1}^{2}}{2\left(1+Q_{1}\right)} M_{1}+\frac{\|A\|\left(T^{2}-t^{2}\right) Q_{1}^{2}}{2\left(1+Q_{1}\right)} M_{1} \\
& =t Q_{1} M_{1}-\frac{\|A\| t^{2} Q_{1}^{2}}{2\left(1+Q_{1}\right)} M_{1}+\frac{\|A\| T^{2} Q_{1}^{2}}{2\left(1+Q_{1}\right)} M_{1}-\frac{\|A\| t^{2} Q_{1}^{2}}{2\left(1+Q_{1}\right)} M_{1} \\
& =t Q_{1} M_{1}-\frac{2\|A\| t^{2} Q_{1}^{2}}{2\left(1+Q_{1}\right)} M_{1}+\frac{\|A\| T^{2} Q_{1}^{2}}{2\left(1+Q_{1}\right)} M_{1} \\
& =\left(t Q_{1}+\frac{\|A\|\left(T^{2}-2 t^{2}\right) Q_{1}^{2}}{2\left(1+Q_{1}\right)}\right) M_{1} \\
& =\alpha_{1}(t) M_{1}
\end{aligned}
$$

مأخوذة 2

لتكن t

ذات دور يساوي T عندئذ المتباينة الآتية:

$\left\|\int_{0}^{t} e^{B(t-s-\tau)}\left[g(\tau, x, y, z)-\int_{0}^{T} \frac{B}{E+e^{B T}} e^{B(T-s-\tau)} g(\tau, x, y, z) d \tau\right] d \tau\right\| \leq \alpha_{2}(t) M_{2}$

$M_{2}=\max _{t}\|g(t, x, y, z)\| \quad$ تتحقق لكل

$$
\alpha_{2}(t)=\left(t Q_{2}+\frac{\|B\|\left(T^{2}-2 t^{2}\right) Q_{2}^{2}}{2\left(1+Q_{2}\right)}\right),
$$

مباشر من المتباينة الآتية: - من

$\left\|\int_{0}^{t} e^{B(t-s-\tau)}\left[g(\tau, x, y, z)-\int_{0}^{T} \frac{B}{E+e^{B T}} e^{B(T-s-\tau)} g(\tau, x, y, z) d \tau\right] d \tau\right\| \leq$ 


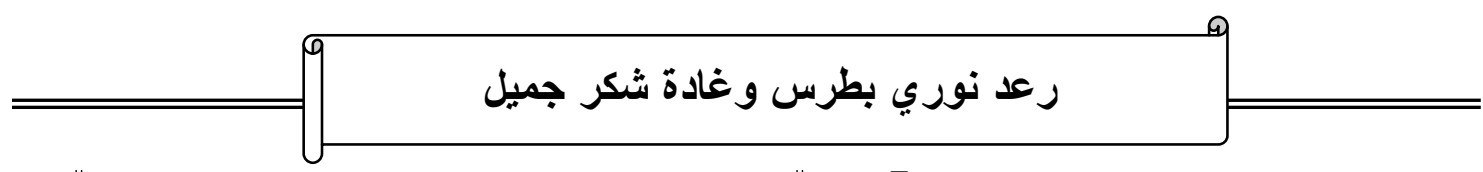

$\leq \int_{0}^{t}\left\|e^{B(t-s-\tau)}\left(E-\frac{B \tau}{E+e^{B T}} e^{B(t-s-\tau)}\right) g(\tau, x, y, z)\right\| d \tau+\int_{t}^{T}\left\|\frac{B \tau}{E+e^{B T}} e^{2 B(t-s-\tau)} g(\tau, x, y, z)\right\| d \tau$ نحصل على $\leq \int_{0}^{t}\left\|\left(E-\frac{B \tau}{E+e^{B T}} e^{B(t-s-\tau)}\right) e^{B(t-s-\tau)}\right\| g(\tau, x, y, z)\left\|d \tau+\int_{t}^{T}\right\| \frac{B \tau}{E+e^{B T}} e^{2 B(t-s-\tau)}\|g(\tau, x, y, z)\| d \tau$ إذن

$$
\begin{aligned}
& \leq \int_{0}^{t}\left(1-\frac{\|B\| \tau}{1+Q_{2}} Q_{2}\right) Q_{2} M_{2} d \tau+\int_{t}^{T} \frac{\|B\| \tau}{1+Q_{2}} Q_{2}^{2} M_{2} d \tau \\
& =t Q_{2} M_{2}-\frac{\|B\| t^{2} Q_{2}^{2}}{2\left(1+Q_{2}\right)} M_{2}+\frac{\|B\|\left(T^{2}-t^{2}\right) Q_{2}^{2}}{2\left(1+Q_{2}\right)} M_{2} \\
& =t Q_{2} M_{2}-\frac{\|B\| t^{2} Q_{2}^{2}}{2\left(1+Q_{2}\right)} M_{2}+\frac{\|B\| T^{2} Q_{2}^{2}}{2\left(1+Q_{2}\right)} M_{2}-\frac{\|B\| t^{2} Q_{2}^{2}}{2\left(1+Q_{2}\right)} M_{2} \\
& =t Q_{2} M_{2}-\frac{2\|B\| t^{2} Q_{2}^{2}}{2\left(1+Q_{2}\right)} M_{2}+\frac{\|B\| T^{2} Q_{2}^{2}}{2\left(1+Q_{2}\right)} M_{2} \\
& =\left(t Q_{2}+\frac{\|B\|\left(T^{2}-2 t^{2}\right) Q_{2}^{2}}{2\left(1+Q_{2}\right)}\right) M_{2} \\
& =\alpha_{2}(t) M_{2}
\end{aligned}
$$

(1) البند الأول: الحل التقريبي الاوري للنظام

مبرهنة 1 :

إذا كان النظام (1) يحقق المتباينات (3) ، (4) ، (5) ، (6) و (8) و الثرط (7)

فإن متتابعات الدو ال:

$$
\begin{array}{r}
x_{m+1}\left(t, x_{0}, y_{0}\right)=x_{0}+\int_{0}^{t} e^{A(t-s-\tau)}\left[f\left(\tau, x_{m}\left(\tau, x_{0}, y_{0}\right), y_{m}\left(\tau, x_{0}, y_{0}\right), z_{m}\left(\tau, x_{0}, y_{0}\right)\right)-\right. \\
\left.-\int_{0}^{T} \frac{A}{E+e^{A T}} e^{A(T-s-\tau)} f\left(\tau, x_{m}\left(\tau, x_{0}, y_{0}\right), y_{m}\left(\tau, x_{0}, y_{0}\right), z_{m}\left(\tau, x_{0}, y_{0}\right)\right) d \tau\right] d \tau
\end{array}
$$$$
x_{0}\left(t, x_{0}\right)=x_{0} e^{A t} \quad, \quad \mathrm{~m}=0,1,2, \ldots
$$

$$
\begin{array}{r}
y_{m+1}\left(t, x_{0}, y_{0}\right)=y_{0}+\int_{0}^{t} e^{B(t-s-\tau)}\left[g\left(\tau, x_{m}\left(\tau, x_{0}, y_{0}\right), y_{m}\left(\tau, x_{0}, y_{0}\right), z_{m}\left(\tau, x_{0}, y_{0}\right)\right)-\right. \\
\left.-\int_{0}^{T} \frac{B}{E+e^{B T}} e^{B(T-s-\tau)} g\left(\tau, x_{m}\left(\tau, x_{0}, y_{0}\right), y_{m}\left(\tau, x_{0}, y_{0}\right), z_{m}\left(\tau, x_{0}, y_{0}\right)\right) d \tau\right] d \tau
\end{array}
$$




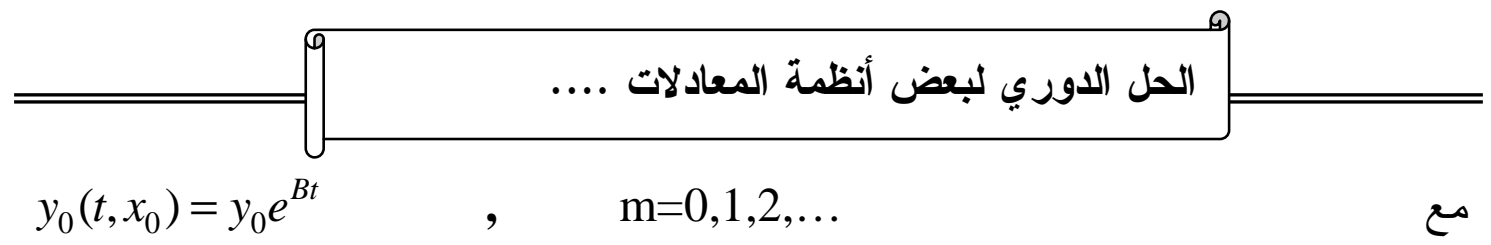

دورية في t ذات دورة تساوي T T ، ومتقاربة بانتظام عندما $\left(t, x_{0}, y_{0}\right) \in R^{1} \times D_{f} \times D_{1 f}$

في كل من الدالة و الدورية في t ذات دورة تساوي T و التي تحقق نظام المعادلات التكاملية: $\begin{aligned} & x\left(t, x_{0}, y_{0}\right)=x_{0}+\int_{0}^{t} e^{A(t-s-\tau)}\left[f\left(\tau, x\left(\tau, x_{0}, y_{0}\right), y\left(\tau, x_{0}, y_{0}\right), z\left(\tau, x_{0}, y_{0}\right)\right)-\right. \\ &\left.-\int_{0}^{T} \frac{A}{E+e^{A T}} e^{A(T-s-\tau)} f\left(\tau, x\left(\tau, x_{0}, y_{0}\right), y\left(\tau, x_{0}, y_{0}\right), z\left(\tau, x_{0}, y_{0}\right)\right) d \tau\right] d \tau\end{aligned}$

$$
\begin{aligned}
y\left(t, x_{0}, y_{0}\right) & =y_{0}+\int_{0}^{t} e^{B(t-s-\tau)}\left[g\left(\tau, x\left(\tau, x_{0}, y_{0}\right), y\left(\tau, x_{0}, y_{0}\right), z\left(\tau, x_{0}, y_{0}\right)\right)-\right. \\
- & \left.\int_{0}^{T} \frac{B}{E+e^{B T}} e^{B(T-s-\tau)} g\left(\tau, x\left(\tau, x_{0}, y_{0}\right), y\left(\tau, x_{0}, y_{0}\right), z\left(\tau, x_{0}, y_{0}\right)\right) d \tau\right] d \tau
\end{aligned}
$$

و الذي هو حل وحيد للنظام (1) بشرط أن يحقق المتباينات الآتية:

$\left\|x_{\infty}\left(t, x_{0}, y_{0}\right)-x_{0}\right\| \leq \frac{\|A\| T^{2} Q_{1}^{2}}{2\left(1+Q_{1}\right)} M_{1}$

$\left\|y_{\infty}\left(t, x_{0}, y_{0}\right)-y_{0}\right\| \leq \frac{\|B\| T^{2} Q_{2}^{2}}{2\left(1+Q_{2}\right)} M_{2}$

$\left(\begin{array}{l}\left\|x_{\infty}\left(t, x_{0}, y_{0}\right)-x_{m}\left(t, x_{0}, y_{0}\right)\right\| \\ \left\|y_{\infty}\left(t, x_{0}, y_{0}\right)-y_{m}\left(t, x_{0}, y_{0}\right)\right\|\end{array}\right) \leq \Lambda_{0}^{m}\left(E-\Lambda_{0}\right)^{-1} V_{0}$

لكل $\Lambda_{0}=\left(\begin{array}{ll}\frac{\|A\| T^{2} Q_{1}^{2}}{2\left(1+Q_{1}\right)}\left(K_{1}+K_{3} \frac{\delta}{\gamma} e^{\gamma T} N_{1}\right) & \frac{\|A\| T^{2} Q_{1}^{2}}{2\left(1+Q_{1}\right)}\left(K_{2}+K_{3} \frac{\delta}{\gamma} e^{\gamma T} N_{2}\right) \\ \frac{\|B\| T^{2} Q_{2}^{2}}{2\left(1+Q_{2}\right)}\left(L_{1}+L_{3} \frac{\delta}{\gamma} e^{\gamma T} N_{1}\right) & \frac{\|B\| T^{2} Q_{2}^{2}}{2\left(1+Q_{2}\right)}\left(L_{2}+L_{3} \frac{\delta}{\gamma} e^{\gamma T} N_{2}\right)\end{array}\right)$, 


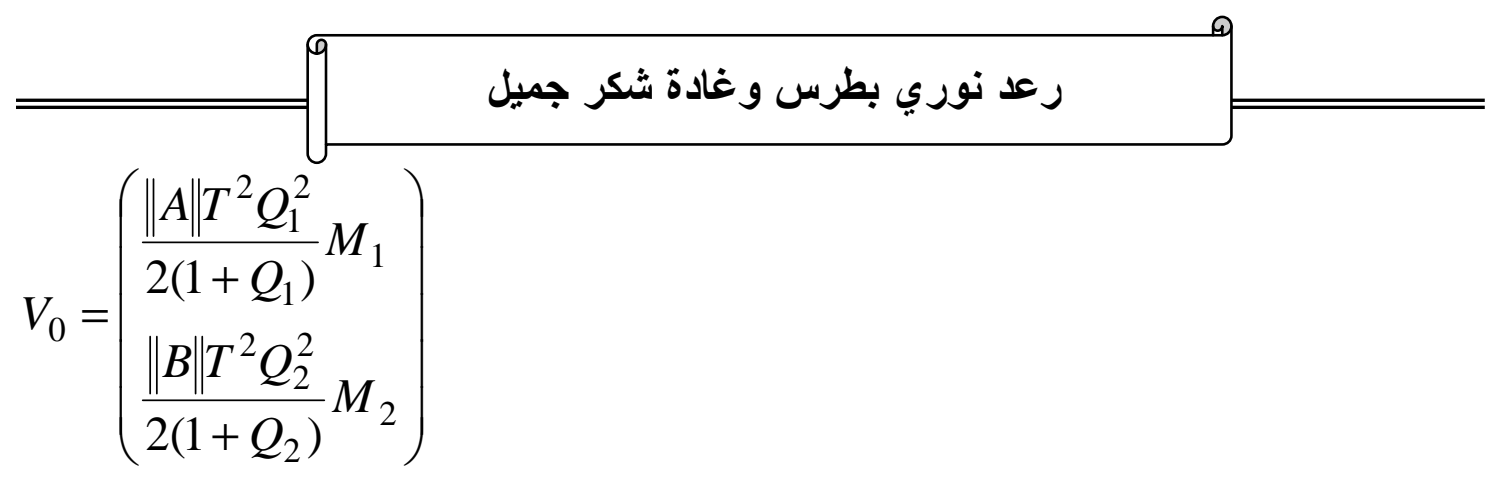

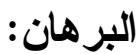

باستخذام المأخوذة 1 وبواسطة المتتابعة (11)، عندما m=0 نحصل على

$$
\begin{array}{r}
\left\|x_{1}\left(t, x_{0}, y_{0}\right)-x_{0}\right\|=\| x_{0}+\int_{0}^{t} e^{A(t-s-\tau)}\left[f\left(\tau, x_{0}\left(\tau, x_{0}, y_{0}\right), y_{0}\left(\tau, x_{0}, y_{0}\right), z_{0}\left(\tau, x_{0}, y_{0}\right)\right)-\right. \\
\left.-\int_{0}^{T} \frac{A}{E+e^{A T}} e^{A(T-s-\tau)} g\left(\tau, x_{0}\left(\tau, x_{0}, y_{0}\right), y_{0}\left(\tau, x_{0}, y_{0}\right), z_{0}\left(\tau, x_{0}, y_{0}\right)\right) d \tau\right] d \tau-x_{0} \| \\
\left\|x_{1}\left(t, x_{0}, y_{0}\right)-x_{0}\right\| \leq\left(t Q_{1}+\frac{\|A\|\left(T^{2}-2 t^{2}\right)}{2\left(1+Q_{1}\right)} Q_{1}^{2}\right) M_{1}=\alpha_{1}(t) M_{1} \quad \ldots . . .(19)
\end{array}
$$

كذلك باستخدام المأخوذة 2 وبو اسطة المتتابعة (12)، عندما m=0 نحصل على $\left\|y_{1}\left(t, x_{0}, y_{0}\right)-y_{0}\right\|=\| y_{0}+\int_{0}^{t} e^{B(t-s-\tau)}\left[g\left(\tau, x_{0}\left(\tau, x_{0}, y_{0}\right), y_{0}\left(\tau, x_{0}, y_{0}\right), z_{0}\left(\tau, x_{0}, y_{0}\right)\right)-\right.$ $\left.-\int_{0}^{T} \frac{B}{E+e^{B T}} e^{B(T-s-\tau)} g\left(\tau, x_{0}\left(\tau, x_{0}, y_{0}\right), y_{0}\left(\tau, x_{0}, y_{0}\right), z_{0}\left(\tau, x_{0}, y_{0}\right)\right) d \tau\right] d \tau-y_{0}$ إذن

$$
\left\|y_{1}\left(t, x_{0}, y_{0}\right)-y_{0}\right\| \leq\left(t Q_{2}+\frac{\|B\|\left(T^{2}-2 t^{2}\right)}{2\left(1+Q_{2}\right)} Q_{2}^{2}\right) M_{2}=\alpha_{2}(t) M_{2}
$$

كنلك من المنباينات (6) ، (19) و (20) نحصل على

$$
\left\|z_{1}\left(\tau, x_{0}, y_{0}\right)-z_{0}(\tau)\right\|=\left\|\int_{-\infty}^{t} u(t, s, \tau) h\left(\tau, x_{1}, y_{1}\right) d \tau-\int_{-\infty}^{t} u(t, s, \tau) h\left(\tau, x_{0}, y_{0}\right) d \tau\right\|
$$

$$
\begin{aligned}
& \leq \int_{-\infty}^{t}\|u(t, s, \tau)\| h\left(\tau, x_{1}, y_{1}\right)-h\left(\tau, x_{0}, y_{0}\right) \| d \tau \\
& \leq \int_{-\infty}^{t} \delta e^{-\gamma(t-s-\tau)}\left[N_{1}\left\|x_{1}-x_{0}\right\|+N_{2}\left\|y_{1}-y_{0}\right\| d \tau\right. \\
& \leq \frac{\delta}{\gamma} e^{\nu T}\left[N_{1} \alpha_{1}(t) M_{1}+N_{2} \alpha_{2}(t) M_{2}\right]
\end{aligned}
$$


$\left\|z_{1}\left(\tau, x_{0}, y_{0}\right)-z_{0}(\tau)\right\| \leq \frac{\delta}{\gamma} e^{\gamma T}\left[N_{1} \frac{\|A\| T^{2} Q_{1}^{2}}{2\left(1+Q_{1}\right)} M_{1}+N_{2} \frac{\|B\| T^{2} Q_{2}^{2}}{2\left(1+Q_{2}\right)} M_{2}\right]$

إذ ان $y_{0} \in D_{1 f}$ ، $x_{0} \in D_{f}$ ، $t \in R^{1} y_{1}\left(t, x_{0}, y_{0}\right) \in D_{1}$ ، $x_{1}\left(t, x_{0}, y_{0}\right) \in D$

$$
z_{0}(\tau)=\int_{-\infty}^{t} u(t, s, \tau) h\left(\tau, x_{0}, y_{0}\right) d \tau \in D_{2 f},
$$

نفترض ان $\left\|x_{m}\left(t, x_{0}, y_{0}\right)-x_{0}\right\| \leq \frac{\|A\| T^{2} Q_{1}^{2}}{2\left(1+Q_{1}\right)} M_{1}$

$\left\|y_{m}\left(t, x_{0}, y_{0}\right)-y_{0}\right\| \leq \frac{\|B\| T^{2} Q_{2}^{2}}{2\left(1+Q_{2}\right)} M_{2}$

$\left\|z_{m}\left(\tau, x_{0}, y_{0}\right)-z_{0}(\tau)\right\| \leq \frac{\delta}{\gamma} e^{\gamma T}\left[N_{1} \frac{\|A\| T^{2} Q_{1}^{2}}{2\left(1+Q_{1}\right)} M_{1}+N_{2} \frac{\|B\| T^{2} Q_{2}^{2}}{2\left(1+Q_{2}\right)} M_{2}\right]$

، $t \in R^{1}$ إذ ان $z_{m}\left(\tau, x_{0}, y_{0}\right) \in D_{2}$ ، $y_{m}\left(t, x_{0}, y_{0}\right) \in D_{1}$ ، $x_{m}\left(t, x_{0}, y_{0}\right) \in D$ - $z_{0}(\tau) \in D_{2 f}$ ، $y_{0} \in D_{1 f}$ ‘ $x_{0} \in D_{f}$

الآن نبر هن ان كلا من المتتابعات (11) و (12) منقاربة بانتظام في المجال (13). بو اسطة المأخوذة (1) ومن المتتابعة (11) عندما m=1 نجد ان: $\left\|x_{2}\left(t, x_{0}, y_{0}\right)-x_{1}\left(t, x_{0}, y_{0}\right)\right\|=\| x_{0}+\int_{0}^{t} e^{A(t-s-\tau)}\left[f\left(\tau, x_{1}\left(\tau, x_{0}, y_{0}\right), y_{1}\left(\tau, x_{0}, y_{0}\right), z_{1}\left(\tau, x_{0}, y_{0}\right)\right)-\right.$ $\left.-\int_{0}^{T} \frac{A}{E+e^{A T}} e^{A(T-s-\tau)} f\left(\tau, x_{1}\left(\tau, x_{0}, y_{0}\right), y_{1}\left(\tau, x_{0}, y_{0}\right), z_{1}\left(\tau, x_{0}, y_{0}\right)\right) d \tau\right] d \tau-$ $-x_{0}-\int_{0}^{t} e^{A(t-s-\tau)}\left[f\left(\tau, x_{0}\left(\tau, x_{0}, y_{0}\right), y_{0}\left(\tau, x_{0}, y_{0}\right), z_{0}\left(\tau, x_{0}, y_{0}\right)\right)+\right.$ $\left.+\int_{0}^{T} \frac{A}{E+e^{A T}} e^{A(T-s-\tau)} f\left(\tau, x_{0}\left(\tau, x_{0}, y_{0}\right), y_{0}\left(\tau, x_{0}, y_{0}\right), z_{0}\left(\tau, x_{0}, y_{0}\right)\right) d \tau\right] d \tau \|$ $\leq \int_{0}^{t}\left(1-\frac{\|A\| \tau}{1+\left\|e^{A T}\right\|}\left\|e^{A(t-s-\tau)}\right\|\left\|e^{A(t-s-\tau)}\right\|\left[K_{1}\left\|x_{1}-x_{0}\right\|+K_{2}\left\|y_{1}-y_{0}\right\|+K_{3}\left\|z_{1}-z_{0}\right\|\right] d \tau+\right.$ $+\int_{t}^{T} \frac{\|A\| \tau}{1+\left\|e^{A T}\right\|}\left\|e^{2 A(t-s-\tau)}\right\|\left[K_{1}\left\|x_{1}-x_{0}\right\|+K_{2}\left\|y_{1}-y_{0}\right\|+K_{3}\left\|z_{1}-z_{0}\right\|\right] d \tau$ 


$$
\begin{aligned}
& \text { لص رعد نوري بطرس وغادة شكر جميل } \\
& \leq \int_{0}^{t}\left(1-\frac{\|A\| \tau}{1+Q_{1}} Q_{1}\right) Q_{1}\left[K_{1}\left\|x_{1}-x_{0}\right\|+K_{2}\left\|y_{1}-y_{0}\right\|+K_{3} \frac{\delta}{\gamma} e^{\lambda T}\left(N_{1}\left\|x_{1}-x_{0}\right\|+N_{2}\left\|y_{1}-y_{0}\right\|\right)\right] d \tau+ \\
& +\int_{t}^{T} \frac{\|A\| \tau}{1+Q_{1}} Q_{1}^{2}\left[K_{1}\left\|x_{1}-x_{0}\right\|+K_{2}\left\|y_{1}-y_{0}\right\|+K_{3} \frac{\delta}{\gamma} e^{\gamma T}\left(N_{1}\left\|x_{1}-x_{0}\right\|+N_{2}\left\|y_{1}-y_{0}\right\|\right)\right] d \tau \\
& \leq\left(t Q_{1}-\frac{\|A\|\left(T^{2}-2 t^{2}\right)}{2\left(1+Q_{1}\right)} Q_{1}^{2}\right)\left[\left(K_{1}+K_{3} \frac{\delta}{\gamma} e^{\gamma T} N_{1}\right) \mid x_{1}-x_{0}\left\|+\left(K_{2}+K_{3} \frac{\delta}{\gamma} e^{\gamma T} N_{2}\right)\right\| y_{1}-y_{0} \|\right] \\
& =\alpha_{1}(t)\left[\left(K_{1}+K_{3} \frac{\delta}{\gamma} e^{\gamma T} N_{1}\right)\left\|x_{1}-x_{0}\right\|+\left(K_{2}+K_{3} \frac{\delta}{\gamma} e^{\gamma T} N_{2}\right)\left\|y_{1}-y_{0}\right\|\right] \\
& \leq \frac{\|A\| T^{2} Q_{1}^{2}}{2\left(1+Q_{1}\right)}\left[\left(K_{1}+K_{3} \frac{\delta}{\gamma} e^{\gamma T} N_{1}\right)\left\|x_{1}-x_{0}\right\|+\left(K_{2}+K_{3} \frac{\delta}{\gamma} e^{\gamma T} N_{2}\right)\left\|y_{1}-y_{0}\right\|\right]
\end{aligned}
$$

$\left\|y_{2}\left(t, x_{0}, y_{0}\right)-y_{1}\left(t, x_{0}, y_{0}\right)\right\| \leq \int_{0}^{t}\left(1-\frac{\|B\| \tau}{1+Q_{2}} Q_{2}\right) Q_{2}\left[L_{1}\left\|x_{1}-x_{0}\right\|+L_{2}\left\|y_{1}-y_{0}\right\|+L_{3}\left\|z_{1}-z_{0}\right\|\right] d \tau+$

$$
+\int_{t}^{T} \frac{\|B\| \tau}{1+Q_{2}} Q_{2}^{2}\left[L_{1}\left\|x_{1}-x_{0}\right\|+L_{2}\left\|y_{1}-y_{0}\right\|+L_{3}\left\|z_{1}-z_{0}\right\|\right] d \tau
$$$$
\leq\left(t Q_{2}-\frac{\|B\|\left(T^{2}-2 t^{2}\right)}{2\left(1+Q_{2}\right)} Q_{2}^{2}\right)\left[\left(L_{1}+L_{3} \frac{\delta}{\gamma} e^{\gamma T} N_{1}\right) \mid x_{1}-x_{0}\left\|+\left(L_{2}+L_{3} \frac{\delta}{\gamma} e^{\gamma T} N_{2}\right)\right\| y_{1}-y_{0} \|\right]
$$$$
=\alpha_{2}(t)\left[\left(L_{1}+L_{3} \frac{\delta}{\gamma} e^{\gamma T} N_{1}\right)\left\|x_{1}-x_{0}\right\|+\left(L_{2}+L_{3} \frac{\delta}{\gamma} e^{\gamma T} N_{2}\right)\left\|y_{1}-y_{0}\right\|\right]
$$$$
\leq \frac{\|B\| T^{2} Q_{2}^{2}}{2\left(1+Q_{2}\right)}\left[\left(L_{1}+L_{3} \frac{\delta}{\gamma} e^{\gamma T} N_{1}\right)\left\|x_{1}-x_{0}\right\|+\left(L_{2}+L_{3} \frac{\delta}{\gamma} e^{\gamma T} N_{2}\right)\left\|y_{1}-y_{0}\right\|\right]
$$

كذلك

$$
\left\|z_{2}\left(\tau, x_{0}, y_{0}\right)-z_{1}\left(\tau, x_{0}, y_{0}\right)\right\| \leq \frac{\delta}{\gamma} e^{\gamma T}\left[N_{1}\left\|x_{2}-x_{1}\right\|+N_{2}\left\|y_{2}-y_{1}\right\|\right]
$$

بو اسطة المأخوذة (1) ومن المتتابعة (11) عندما m=2 نحصل على: $\left\|x_{3}\left(t, x_{0}, y_{0}\right)-x_{2}\left(t, x_{0}, y_{0}\right)\right\| \leq \int_{0}^{t}\left(1-\frac{\|A\| \tau}{1+Q_{1}} Q_{1}\right) Q_{1}\left[K_{1}\left\|x_{2}-x_{1}\right\|+K_{2}\left\|y_{2}-y_{1}\right\|+K_{3}\left\|z_{2}-z_{1}\right\| d \tau+\right.$

$$
+\int_{t}^{T} \frac{\| A \mid \tau}{1+Q_{1}} Q_{1}^{2}\left[K_{1}\left\|x_{2}-x_{1}\right\|+K_{2}\left\|y_{2}-y_{1}\right\|+K_{3}\left\|z_{2}-z_{1}\right\|\right] d \tau
$$




$$
\begin{aligned}
& \text { لص الحل الدوري لبعض أنظمة المعادلات ..... } \\
& \leq \alpha_{1}(t)\left[\left(K_{1}+K_{3} \frac{\delta}{\gamma} e^{\gamma T} N_{1}\right)\left\|x_{2}-x_{1}\right\|+\left(K_{2}+K_{3} \frac{\delta}{\gamma} e^{\gamma T} N_{2}\right)\left\|y_{2}-y_{1}\right\|\right] \\
& \leq \frac{\|A\| T^{2} Q_{1}^{2}}{2\left(1+Q_{1}\right)}\left[\left(K_{1}+K_{3} \frac{\delta}{\gamma} e^{\gamma T} N_{1}\right)\left|x_{2}-x_{1}\left\|+\left(K_{2}+K_{3} \frac{\delta}{\gamma} e^{\gamma T} N_{2}\right) \mid y_{2}-y_{1}\right\|\right]\right. \\
& \text { وبو اسطة المأخوذة (2) ومن المتتابعة (12) عندما m=2 ينتج لدينا: } \\
& \left\|y_{3}\left(t, x_{0}, y_{0}\right)-y_{2}\left(t, x_{0}, y_{0}\right)\right\| \leq \int_{0}^{t}\left(1-\frac{\|B\| \tau}{1+Q_{2}} Q_{2}\right) Q_{2}\left[L_{1}\left\|x_{2}-x_{1}\right\|+L_{2}\left\|y_{2}-y_{1}\right\|+L_{3}\left\|z_{2}-z_{1}\right\| d \tau+\right. \\
& +\int_{t}^{T} \frac{\|B\| \tau}{1+Q_{2}} Q_{2}^{2}\left[L_{1}\left\|x_{2}-x_{1}\right\|+L_{2}\left\|y_{2}-y_{1}\right\|+L_{3}\left\|z_{2}-z_{1}\right\|\right] d \tau \\
& \leq \alpha_{2}(t)\left[\left(L_{1}+L_{3} \frac{\delta}{\gamma} e^{\gamma T} N_{1}\right)\left\|x_{2}-x_{1}\right\|+\left(L_{2}+L_{3} \frac{\delta}{\gamma} e^{\gamma T} N_{2}\right)\left\|y_{2}-y_{1}\right\|\right] \\
& \leq \frac{\|B\| T^{2} Q_{2}^{2}}{2\left(1+Q_{2}\right)}\left[\left(L_{1}+L_{3} \frac{\delta}{\gamma} e^{\gamma T} N_{1}\right)\left\|x_{2}-x_{1}\right\|+\left(L_{2}+L_{3} \frac{\delta}{\gamma} e^{\gamma T} N_{2}\right)\left\|y_{2}-y_{1}\right\|\right]
\end{aligned}
$$

كذللك

$$
\left\|z_{3}\left(\tau, x_{0}, y_{0}\right)-z_{2}\left(\tau, x_{0}, y_{0}\right)\right\| \leq \frac{\delta}{\gamma} e^{\gamma T}\left[N_{1}\left\|x_{3}-x_{2}\right\|+N_{2}\left\|y_{3}-y_{2}\right\|\right]
$$

وبو اسطة الاستقر اء الرياضي ينتج لدينا

$$
\left\|x_{m+1}\left(t, x_{0}, y_{0}\right)-x_{m}\left(t, x_{0}, y_{0}\right)\right\| \leq \frac{\| A T^{2} Q_{1}^{2}}{2\left(1+Q_{1}\right)}\left[\left(K_{1}+K_{3} \frac{\delta}{\gamma} e^{\eta T} N_{1}\right) x_{m}-x_{m-1}\left\|+\left(K_{2}+K_{3} \frac{\delta}{\gamma} e^{\not T} N_{2}\right) y_{m}-y_{m-1}\right\|\right]
$$

$$
\left\|y_{m+1}\left(t, x_{0}, y_{0}\right)-y_{m}\left(t, x_{0}, y_{0}\right)\right\| \leq \frac{\|B\| T^{2} Q_{2}^{2}}{2\left(1+Q_{2}\right)}\left[\left(L_{1}+L_{3} \frac{\delta}{\gamma} e^{\not T} N_{1}\right) x_{m}-x_{m-1}\left\|+\left(L_{2}+L_{3} \frac{\delta}{\gamma} e^{\not T} N_{2}\right) y_{m}-y_{m-1}\right\|\right]
$$

نعيد كتابة المتباينتين (22) و (23) بشكل متجهات و على النحو الآتي:

$$
V_{m+1}\left(t, x_{0}, y_{0}\right) \leq \Lambda(t) V_{m}\left(t, x_{0}, y_{0}\right)
$$

$$
V_{m+1}\left(t, x_{0}, y_{0}\right)=\left(\begin{array}{l}
\left\|x_{m+1}\left(t, x_{0}, y_{0}\right)-x_{m}\left(t, x_{0}, y_{0}\right)\right\| \\
\left\|y_{m+1}\left(t, x_{0}, y_{0}\right)-y_{m}\left(t, x_{0}, y_{0}\right)\right\|
\end{array}\right)
$$




$$
\begin{aligned}
& \hline(t)=\left(\begin{array}{ll}
\frac{\|A\| t^{2} Q_{1}^{2}}{2\left(1+Q_{1}\right)}\left(K_{1}+K_{3} \frac{\delta}{\gamma} e^{\gamma T} N_{1}\right) & \frac{\|A\| t^{2} Q_{1}^{2}}{2\left(1+Q_{1}\right)}\left(K_{2}+K_{3} \frac{\delta}{\gamma} e^{\gamma T} N_{2}\right) \\
\frac{\|B\| t^{2} Q_{2}^{2}}{2\left(1+Q_{2}\right)}\left(L_{1}+L_{3} \frac{\delta}{\gamma} e^{\gamma / T} N_{1}\right) & \frac{\|B\| t^{2} Q_{2}^{2}}{2\left(1+Q_{2}\right)}\left(L_{2}+L_{3} \frac{\delta}{\gamma} e^{\gamma T} N_{2}\right)
\end{array}\right), \\
& V_{m}\left(t, x_{0}, y_{0}\right)=\left(\begin{array}{l}
\left\|x_{m}\left(t, x_{0}, y_{0}\right)-x_{m-1}\left(t, x_{0}, y_{0}\right)\right\| \\
\left\|y_{m}\left(t, x_{0}, y_{0}\right)-y_{m-1}\left(t, x_{0}, y_{0}\right)\right\|
\end{array}\right)
\end{aligned}
$$

و هذا يؤدي من المتباينة (24) إلى ان

$V_{m+1}(t) \leq \Lambda_{0} V_{m}(t)$

$\Lambda_{0}=\max _{t \in[0, T]} \Lambda(t)$

بتكر ار المتباينة (25) نحصل على

$$
V_{m+1}(t) \leq \Lambda_{0}^{m} V_{m}(t)
$$

$V_{0}=\left(\begin{array}{l}\frac{\|A\| T^{2} Q_{1}^{2}}{2\left(1+Q_{1}\right)} M_{1} \\ \frac{\|B\| T^{2} Q_{2}^{2}}{2\left(1+Q_{2}\right)} M_{2}\end{array}\right)$

$\sum_{i=1}^{m} V_{i} \leq \sum_{i=1}^{m} \Lambda_{0}^{i-1} V_{0}$

وبما ان المصفوفة $\Lambda_{0}$ لها قيم ذاتية

$$
\begin{aligned}
\lambda_{\max }\left(\Lambda_{0}\right) & =\frac{1}{2}\left[\frac{\|A\| T^{2} Q_{1}^{2}}{2\left(1+Q_{1}\right)}\left(K_{1}+K_{3} \frac{\delta}{\gamma} e^{\gamma T} N_{1}\right)+\frac{\|B\| T^{2} Q_{2}^{2}}{2\left(1+Q_{2}\right)}\left(L_{2}+L_{3} \frac{\delta}{\gamma} e^{\gamma T} N_{2}\right)\right] \mp \\
& \mp \frac{1}{2}\left(\left[\frac{\|A\| T^{2} Q_{1}^{2}}{2\left(1+Q_{1}\right)}\left(K_{1}+K_{3} \frac{\delta}{\gamma} e^{\gamma T} N_{1}\right)-\frac{\|B\| T^{2} Q_{2}^{2}}{2\left(1+Q_{2}\right)}\left(L_{2}+L_{3} \frac{\delta}{\gamma} e^{\gamma T} N_{2}\right)\right]^{2}+\right.
\end{aligned}
$$

$\left.+\frac{\| A \mid T^{2} Q_{1}^{2}}{\left(1+Q_{1}\right)} \frac{\|B\| T^{2} Q_{2}^{2}}{\left(1+Q_{2}\right)}\left[\left(K_{1}+K_{3} \frac{\delta}{\gamma} e^{\gamma T} N_{1}\right)\left(L_{2}+L_{3} \frac{\delta}{\gamma} e^{\gamma T} N_{2}\right)-\left(K_{2}+K_{3} \frac{\delta}{\gamma} e^{\gamma T} N_{2}\right)\left(L_{1}+L_{3} \frac{\delta}{\gamma} e^{\gamma T} N_{1}\right)\right]\right)^{\frac{1}{2}}$

فان هذا يؤدي إلى ان متتابعة الدو ال (11) و (12) متقاربة بانتظام في المجال (13) بمعنـى 
$\operatorname{Lim}_{m \rightarrow \infty} \sum_{i=1}^{m} \Lambda_{0}^{i-1} V_{0}=\sum_{i=1}^{\infty} \Lambda_{0}^{i-1} V_{0}=\left(E-\Lambda_{0}\right)^{-1} V_{0}$

إذ ان E Eفوفة الوحدة.

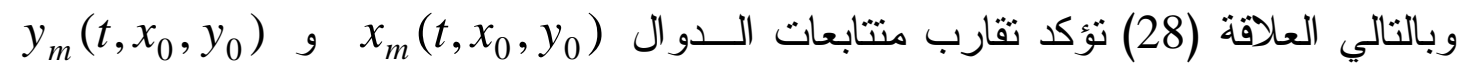
في المجال (13). نفرض ان

$\left.\begin{array}{l}\operatorname{Lim}_{m \rightarrow \infty} x_{m}\left(t, x_{0}\right)=x_{\infty}\left(t, x_{0}\right) \\ \operatorname{Lim}_{m \rightarrow \infty} y_{m}\left(t, x_{0}\right)=y_{\infty}\left(t, x_{0}\right)\end{array}\right\}$

بما أن منتابعة كل من الدالتين دور يساوي T فإن نهاية كل منهما دورية في t ذات دور يساوي T ومن ثم فانن $x_{\infty}\left(t, x_{0}, y_{0}\right)=x\left(t, x_{0}, y_{0}\right) \quad, \quad y_{\infty}\left(t, x_{0}, y_{0}\right)=y\left(t, x_{0}, y_{0}\right)$

فضلا عن ذلك باستخدام العلاقة (29) فإن المتباينة الآتية:

$\left(\begin{array}{l}\left\|x_{\infty}\left(t, x_{0}, y_{0}\right)-x_{m}\left(t, x_{0}, y_{0}\right)\right\| \\ \left\|y_{\infty}\left(t, x_{0}, y_{0}\right)-y_{m}\left(t, x_{0}, y_{0}\right)\right\|\end{array}\right) \leq \Lambda_{0}^{m}\left(E-\Lambda_{0}\right)^{-1} V_{0}$

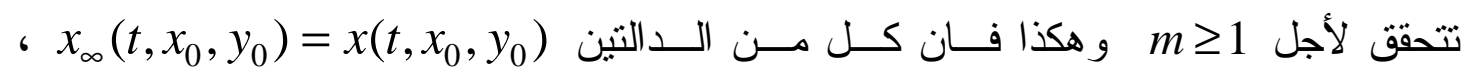
$y_{\infty}\left(t, x_{0}, y_{0}\right)=y\left(t, x_{0}, y_{0}\right)$ البند الثاني: وحدانية الحل

مبرهنة 2 :

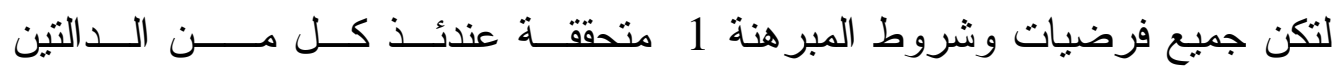

(2) هو حل وحيد للنظام (1) $y\left(t, x_{0}, y_{0}, y_{0}\right)$ البرهان: نفرض ان $\hat{x}\left(t, x_{0}, y_{0}\right)=x_{0}+\int_{0}^{t} e^{A(t-s-\tau)}\left[f\left(\tau, \hat{x}\left(\tau, x_{0}, y_{0}\right), \hat{y}\left(\tau, x_{0}, y_{0}\right), \hat{z}\left(\tau, x_{0}, y_{0}\right)\right)-\right.$ $\left.-\int_{0}^{T} \frac{A}{E+e^{A T}} e^{A(T-s-\tau)} f\left(\tau, \hat{x}\left(\tau, x_{0}, y_{0}\right), \hat{y}\left(\tau, x_{0}, y_{0}\right), \hat{z}\left(\tau, x_{0}, y_{0}\right)\right) d \tau\right] d \tau$ $\hat{y}\left(t, x_{0}, y_{0}\right)=y_{0}+\int_{0}^{t} e^{B(t-s-\tau)}\left[g\left(\tau, \hat{x}\left(\tau, x_{0}, y_{0}\right), \hat{y}\left(\tau, x_{0}, y_{0}\right), \hat{z}\left(\tau, x_{0}, y_{0}\right)\right)-\right.$ $\left.-\int_{0}^{T} \frac{B}{E+e^{B T}} e^{B(T-s-\tau)} g\left(\tau, \hat{x}\left(\tau, x_{0}, y_{0}\right), \hat{y}\left(\tau, x_{0}, y_{0}\right), \hat{z}\left(\tau, x_{0}, y_{0}\right)\right) d \tau\right] d \tau$ 


$$
\begin{aligned}
& \text { لص رعد نوري بطرس وغادة شكر جميل }
\end{aligned}
$$

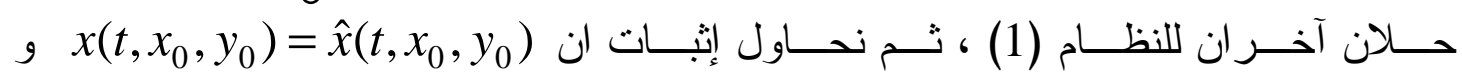

$$
\begin{aligned}
& \text { ( و لإثبات ذللك نحتاج إلى إثبات صحة المتباينة الآثية: } y\left(t, x_{0}, y_{0}\right)=\hat{y}\left(t, x_{0}, y_{0}\right) \\
& \left(\begin{array}{l}
\left\|\hat{x}\left(t, x_{0}, y_{0}\right)-x_{m}\left(t, x_{0}, y_{0}\right)\right\| \\
\left\|\hat{y}\left(t, x_{0}, y_{0}\right)-y_{m}\left(t, x_{0}, y_{0}\right)\right\|
\end{array}\right) \leq \Lambda_{0}\left(\begin{array}{l}
\left\|\hat{x}\left(t, x_{0}, y_{0}\right)-x_{m-1}\left(t, x_{0}, y_{0}\right)\right\| \\
\left\|\hat{y}\left(t, x_{0}, y_{0}\right)-y_{m-1}\left(t, x_{0}, y_{0}\right)\right\|
\end{array}\right) \\
& \text { - } M_{2}^{*}=\max _{t \in[0, T]}\|g(t, x, y, z)\| \quad \text { ، } M_{1}^{*}=\max _{t \in[0, T]}\|f(t, x, y, z)\| \stackrel{ث}{*} \\
& \text { سوف نتبت المتباينة أعلاه باستخدام طريقة الاستقر اء الرياضي. } \\
& \text { عندما m=0 نحصل على } \\
& \left\|\hat{x}\left(t, x_{0}, y_{0}\right)-x_{0}\right\|=\| x_{0}+\int_{0}^{t} e^{A(t-s-\tau)}\left[f\left(\tau, \hat{x}\left(\tau, x_{0}, y_{0}\right), \hat{y}\left(\tau, x_{0}, y_{0}\right), \hat{z}\left(\tau, x_{0}, y_{0}\right)\right)-\right. \\
& \left.-\int_{0}^{T} \frac{A}{E+e^{A T}} e^{A(T-s-\tau)} f\left(\tau, \hat{x}\left(\tau, x_{0}, y_{0}\right), \hat{y}\left(\tau, x_{0}, y_{0}\right), \hat{z}\left(\tau, x_{0}, y_{0}\right)\right) d \tau\right] d \tau-x_{0} \| \\
& \leq \alpha_{1}(t) M_{1}^{*} \leq \frac{\|A\| T^{2} Q_{1}^{2}}{2\left(1+Q_{1}\right)} M_{1}^{*}
\end{aligned}
$$$$
\left\|\hat{y}\left(t, x_{0}, y_{0}\right)-y_{0}\right\|=\| y_{0}+\int_{0}^{t} e^{B(t-s-\tau)}\left[g\left(\tau, \hat{x}\left(\tau, x_{0}, y_{0}\right), \hat{y}\left(\tau, x_{0}, y_{0}\right), \hat{z}\left(\tau, x_{0}, y_{0}\right)\right)-\right.
$$$$
\left.-\int_{0}^{T} \frac{B}{E+e^{B T}} e^{B(T-s-\tau)} g\left(\tau, \hat{x}\left(\tau, x_{0}, y_{0}\right), \hat{y}\left(\tau, x_{0}, y_{0}\right), \hat{z}\left(\tau, x_{0}, y_{0}\right)\right) d \tau\right] d \tau-y_{0} \|
$$$$
\leq \alpha_{2}(t) M_{2}^{*} \leq \frac{\|B\| T^{2} Q_{2}^{2}}{2\left(1+Q_{2}\right)} M_{2}^{*}
$$$$
\left\|\hat{z}\left(\tau, x_{0}, y_{0}\right)-z_{0}(\tau)\right\|=\left\|\int_{-\infty}^{t} u(t, s, \tau) h(\tau, \hat{x}, \hat{y}) d \tau-\int_{-\infty}^{t} u(t, s, \tau) h\left(\tau, x_{0}, y_{0}\right) d \tau\right\|
$$$$
\leq \frac{\delta}{\gamma} e^{\gamma T}\left[N_{1}\left\|\hat{x}-x_{0}\right\|+N_{2}\left\|\hat{y}-y_{0}\right\|\right]
$$

عندما m=1 نحصل على

$\left\|\hat{x}\left(t, x_{0}, y_{0}\right)-x_{1}\left(t, x_{0}, y_{0}\right)\right\| \leq \frac{\| A T^{2} Q_{1}^{2}}{2\left(1+Q_{1}\right)}\left[\left(K_{1}+K_{3} \frac{\delta}{\gamma} e^{\gamma T} N_{1}\right) \hat{x}-x_{0}\left\|+\left(K_{2}+K_{3} \frac{\delta}{\gamma} e^{\gamma T} N_{2}\right) \hat{y}-y_{0}\right\|\right]$ $\left\|\hat{y}\left(t, x_{0}, y_{0}\right)-y_{1}\left(t, x_{0}, y_{0}\right)\right\| \leq \frac{\|B\| T^{2} Q_{2}^{2}}{2\left(1+Q_{2}\right)}\left[\left(L_{1}+L_{3} \frac{\delta}{\gamma} e^{\gamma T} N_{1}\right) \mid \hat{x}-x_{0}\left\|+\left(L_{2}+L_{3} \frac{\delta}{\gamma} e^{\gamma T} N_{2}\right) \hat{y}-y_{0}\right\|\right]$ $\left\|\hat{z}\left(\tau, x_{0}, y_{0}\right)-z_{1}\left(\tau, x_{0}, y_{0}\right)\right\| \leq \frac{\delta}{\gamma} e^{\gamma T}\left[N_{1}\left\|\hat{x}-x_{1}\right\|+N_{2}\left\|\hat{y}-y_{1}\right\|\right]$ 


$$
\begin{aligned}
& \text { صل الحل الدوري لبعض أنظمة المعادلات .... } \\
& \text { عندما m=2 نحصل على } \\
& \left\|\hat{x}\left(t, x_{0}, y_{0}\right)-x_{2}\left(t, x_{0}, y_{0}\right)\right\| \leq \frac{\| A T^{2} Q_{1}^{2}}{2\left(1+Q_{1}\right)}\left[\left(K_{1}+K_{3} \frac{\delta}{\gamma} e^{\gamma T} N_{1}\right) \mid \hat{x}-x_{1}\left\|+\left(K_{2}+K_{3} \frac{\delta}{\gamma} e^{\eta T} N_{2}\right) \hat{y}-y_{1}\right\|\right] \\
& \left\|\hat{y}\left(t, x_{0}, y_{0}\right)-y_{2}\left(t, x_{0}, y_{0}\right)\right\| \leq \frac{\| B \mid T^{2} Q_{2}^{2}}{2\left(1+Q_{2}\right)}\left[\left(L_{1}+L_{3} \frac{\delta}{\gamma} e^{\eta T} N_{1}\right) \hat{x}-x_{1}\left\|+\left(L_{2}+L_{3} \frac{\delta}{\gamma} e^{{ }^{T T}} N_{2}\right) \mid \hat{y}-y_{1}\right\|\right] \\
& \left\|\hat{z}\left(\tau, x_{0}, y_{0}\right)-z_{2}\left(\tau, x_{0}, y_{0}\right)\right\| \leq \frac{\delta}{\gamma} e^{\gamma T}\left[N_{1}\left\|\hat{x}-x_{2}\right\|+N_{2}\left\|\hat{y}-y_{2}\right\|\right] \\
& \text { إذن المتباينة صحيحة عندما .m=0,1,2 . } \\
& \text { نفرض صحة المتباينة عندما m=p-1 ، إذن } \\
& \left(\begin{array}{l}
\left\|\hat{x}\left(t, x_{0}, y_{0}\right)-x_{p-1}\left(t, x_{0}, y_{0}\right)\right\| \\
\left\|\hat{y}\left(t, x_{0}, y_{0}\right)-y_{p-1}\left(t, x_{0}, y_{0}\right)\right\|
\end{array}\right) \leq \Lambda_{0}\left(\begin{array}{l}
\left\|\hat{x}\left(t, x_{0}, y_{0}\right)-x_{p-2}\left(t, x_{0}, y_{0}\right)\right\| \\
\left\|\hat{y}\left(t, x_{0}, y_{0}\right)-y_{p-2}\left(t, x_{0}, y_{0}\right)\right\|
\end{array}\right) \\
& \text { إذن سوف نثبت صحة المتباينة عندما m=p. } \\
& \left\|\hat{x}\left(t, x_{0}, y_{0}\right)-x_{p}\left(t, x_{0}, y_{0}\right)\right\| \leq \frac{\| A T^{2} Q_{1}^{2}}{2\left(1+Q_{1}\right)}\left[\left(K_{1}+K_{3} \frac{\delta}{\gamma} e^{2 T} N_{1}\right) \hat{x}-x_{p-1}\left\|+\left(K_{2}+K_{3} \frac{\delta}{\gamma} e^{2 T} N_{2}\right) \hat{y}-y_{p-1}\right\|\right] \\
& \left\|\hat{y}\left(t, x_{0}, y_{0}\right)-y_{p}\left(t, x_{0}, y_{0}\right)\right\| \leq \frac{\| B \mid T^{2} Q_{2}^{2}}{2\left(1+Q_{2}\right)}\left[\left(L_{1}+L_{3} \frac{\delta}{\gamma} e^{\lambda T} N_{1}\right) \mid \hat{x}-x_{p-1}\left\|+\left(L_{2}+L_{3} \frac{\delta}{\gamma} e^{\lambda T} N_{2}\right)\right\| \hat{y}-y_{p-1} \|\right]
\end{aligned}
$$

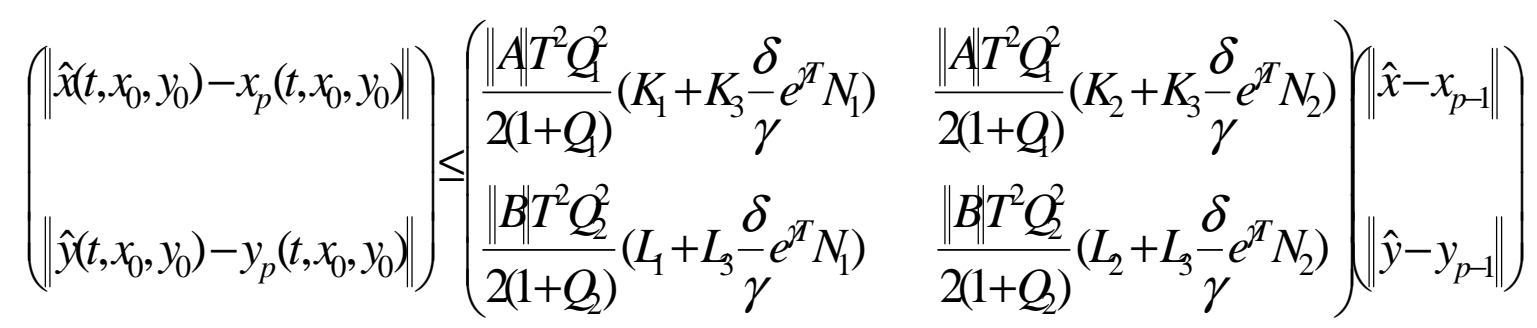

$$
\begin{aligned}
& \left(\begin{array}{l}
\left\|\hat{x}\left(t, x_{0}, y_{0}\right)-x_{p}\left(t, x_{0}, y_{0}\right)\right\| \\
\left\|\hat{y}\left(t, x_{0}, y_{0}\right)-y_{p}\left(t, x_{0}, y_{0}\right)\right\|
\end{array}\right) \leq \Lambda_{0}\left(\begin{array}{l}
\left\|\hat{x}\left(t, x_{0}, y_{0}\right)-x_{p-1}\left(t, x_{0}, y_{0}\right)\right\| \\
\left\|\hat{y}\left(t, x_{0}, y_{0}\right)-y_{p-1}\left(t, x_{0}, y_{0}\right)\right\|
\end{array}\right)
\end{aligned}
$$

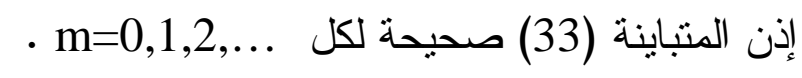

$$
\begin{aligned}
& \text { وبتكرار المنباينة (33) نحصل على إلى } \\
& \left(\begin{array}{l}
\left\|\hat{x}\left(t, x_{0}, y_{0}\right)-x_{m}\left(t, x_{0}, y_{0}\right)\right\| \\
\left\|\hat{y}\left(t, x_{0}, y_{0}\right)-y_{m}\left(t, x_{0}, y_{0}\right)\right\|
\end{array}\right) \leq \Lambda_{0}^{m}\left(\begin{array}{l}
\left\|\hat{x}\left(t, x_{0}, y_{0}\right)-x_{m-1}\left(t, x_{0}, y_{0}\right)\right\| \\
\left\|\hat{y}\left(t, x_{0}, y_{0}\right)-y_{m-1}\left(t, x_{0}, y_{0}\right)\right\|
\end{array}\right)
\end{aligned}
$$

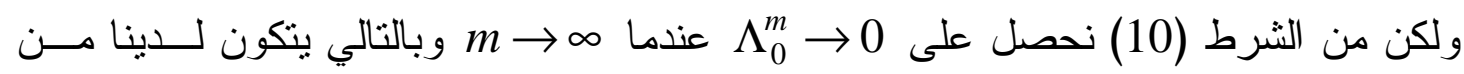

$$
\begin{aligned}
& \text { المتباينة الأخيرة ان } \\
& \text { آخر ان الحلين }
\end{aligned}
$$




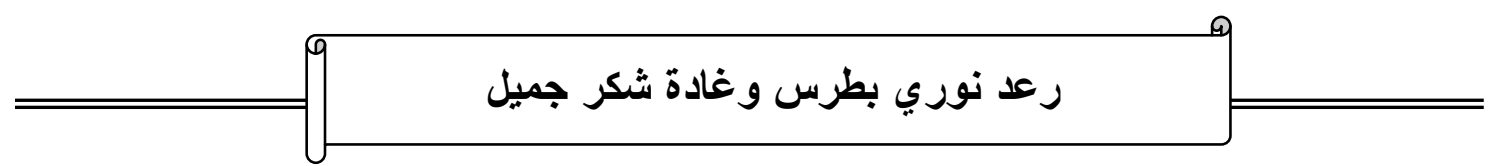

البند الثالث: وجود الحل الاوري للنظام (1)

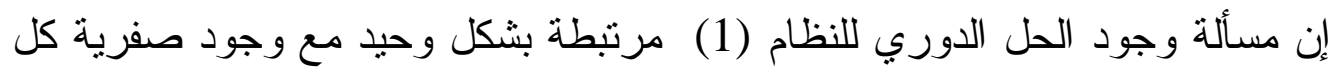
من الدالتين $\Delta\left(0, x_{0}, y_{0}\right)=\int_{0}^{T} \frac{A}{E+e^{A T}} e^{A(T-s-\tau)} f\left(\tau, x_{\infty}\left(\tau, x_{0}, y_{0}\right), y_{\infty}\left(\tau, x_{0}, y_{0}\right), z_{\infty}\left(\tau, x_{0}, y_{0}\right)\right) d \tau$ $\Delta^{*}\left(0, x_{0}, y_{0}\right)=\int_{0}^{T} \frac{B}{E+e^{B T}} e^{B(T-s-\tau)} g\left(\tau, x_{\infty}\left(\tau, x_{0}, y_{0}\right), y_{\infty}\left(\tau, x_{0}, y_{0}\right), z_{\infty}\left(\tau, x_{0}, y_{0}\right)\right) d \tau$

حيث أن و هذه الدو ال لا يمكن إيجادها إلا بطريقة التقريبات المتتالية و لاسيما من متتــابعتي الــالتين

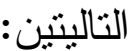
$\Delta_{m}\left(0, x_{0}, y_{0}\right)=\int_{0}^{T} \frac{A}{E+e^{A T}} e^{A(T-s-\tau)} f\left(\tau, x_{m}\left(\tau, x_{0}, y_{0}\right), y_{m}\left(\tau, x_{0}, y_{0}\right), z_{m}\left(\tau, x_{0}, y_{0}\right)\right) d \tau$ $\Delta_{m}^{*}\left(0, x_{0}, y_{0}\right)=\int_{0}^{T} \frac{B}{E+e^{B T}} e^{B(T-s-\tau)} g\left(\tau, x_{m}\left(\tau, x_{0}, y_{0}\right), y_{m}\left(\tau, x_{0}, y_{0}\right), z_{m}\left(\tau, x_{0}, y_{0}\right)\right) d \tau$

$$
\text { . } \mathrm{m}=0,1,2, \ldots \text { حيث }
$$

مبرهنة 3 : مبر

إذا كانت فرضيات وشروط المبرهنة 1 معطاة فإن المنباينة الآتية: $\left(\left\|\Delta\left(t, x_{0}, y_{0}\right)-\Delta_{n}\left(t, x_{0}, y_{0}\right)\right\|\right) \leq\left(\begin{array}{ll}\frac{\| A \mid T Q}{1+Q}\left(K_{1}+K_{3} \frac{\delta}{\gamma} e^{T T} N_{1}\right) & \frac{\| A T Q}{1+Q}\left(K_{2}+K_{3}-\frac{\delta}{\gamma} e^{T T} N_{2}\right) \\ \left\|\Delta^{*}\left(t, x_{0}, y_{0}\right)-\Delta_{n}^{*}\left(t, x_{0}, y_{0}\right)\right\|\end{array}\right) \leq \Lambda_{0}^{m}\left(E-\Lambda_{0}\right)^{-1} V_{0}$

• تتحقق لكل $y_{0} \in D_{1 f}$ ، $x_{0} \in D_{f}$ ، $t \in[0, T] ، m \geq 0$ ،

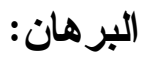
بو اسطة المعادلات (35) ، (36) يكون لدينا 


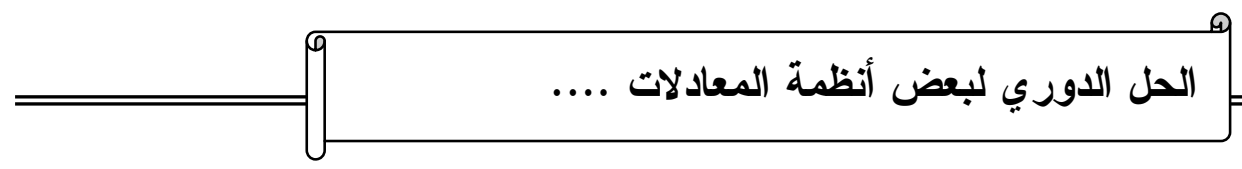

$$
\begin{aligned}
\left\|\Delta\left(0, x_{0}, y_{0}\right)-\Delta_{m}\left(0, x_{0}, y_{0}\right)\right\| & =\| \int_{0}^{T} \frac{A}{E+e^{A T}} e^{A(T-s-\tau)} f\left(\tau, x_{\infty}\left(\tau, x_{0}, y_{0}\right), y_{\infty}\left(\tau, x_{0}, y_{0}\right), z_{\infty}\left(\tau, x_{0}, y_{0}\right)\right) d \tau- \\
& -\int_{0}^{T} \frac{A}{E+e^{A T}} e^{A(T-s-\tau)} f\left(\tau, x_{m}\left(\tau, x_{0}, y_{0}\right), y_{m}\left(\tau, x_{0}, y_{0}\right), z_{m}\left(\tau, x_{0}, y_{0}\right)\right) d \tau \| \\
\leq & \int_{0}^{T} \frac{\|A\|}{1+Q_{1}} Q_{1}\left[K_{1}\left\|x_{\infty}-x_{m}\right\|+K_{2}\left\|y_{\infty}-y_{m}\right\|+K_{3} \frac{\delta}{\gamma} e^{\gamma T}\left(N_{1}\left\|x_{\infty}-x_{m}\right\|+N_{2}\left\|y_{\infty}-y_{m}\right\|\right)\right] d \tau
\end{aligned}
$$$$
\left\|\Delta\left(0, x_{0}, y_{0}\right)-\Delta_{m}\left(0, x_{0}, y_{0}\right)\right\| \leq \frac{\|A\| T Q_{1}}{1+Q_{1}}\left[\left(K_{1}+K_{3} \frac{\delta}{\gamma} e^{\gamma T} N_{1}\right) \mid x_{\infty}-x_{m}\left\|+\left(K_{2}+K_{3} \frac{\delta}{\gamma} e^{\gamma T} N_{2}\right)\right\| y_{\infty}-y_{m} \|\right]
$$

وبالطريقة نفسها وبو اسطة (36) و (38) نجد ان:

$$
\begin{aligned}
\left\|\Delta^{*}\left(0, x_{0}, y_{0}\right)-\Delta_{m}^{*}\left(0, x_{0}, y_{0}\right)\right\| & =\int_{0}^{T} \frac{B}{E+e^{B T}} e^{B(T-s-\tau)} g\left(\tau, x_{\infty}\left(\tau, x_{0}, y_{0}\right), y_{\infty}\left(\tau, x_{0}, y_{0}\right), z_{\infty}\left(\tau, x_{0}, y_{0}\right)\right) d \tau- \\
& -\int_{0}^{T} \frac{B}{E+e^{B T}} e^{B(T-s-\tau)} g\left(\tau, x_{m}\left(\tau, x_{0}, y_{0}\right), y_{m}\left(\tau, x_{0}, y_{0}\right), z_{m}\left(\tau, x_{0}, y_{0}\right)\right) d \tau \| \\
\leq & \int_{0}^{T} \frac{\|B\|}{1+Q_{2}} Q_{2}\left[L_{1}\left\|x_{\infty}-x_{m}\right\|+L_{2}\left\|y_{\infty}-y_{m}\right\|+L_{3} \frac{\delta}{\gamma} e^{\gamma T}\left(N_{1}\left\|x_{\infty}-x_{m}\right\|+N_{2}\left\|y_{\infty}-y_{m}\right\|\right)\right] d \tau
\end{aligned}
$$

$\left\|\Delta^{*}\left(0, x_{0}, y_{0}\right)-\Delta_{m}^{*}\left(0, x_{0}, y_{0}\right)\right\| \leq \frac{\|B\| T Q_{2}}{1+Q_{2}}\left[\left(L_{1}+L_{3} \frac{\delta}{\gamma} e^{\gamma T} N_{1}\right)\left|x_{\infty}-x_{m}\left\|+\left(L_{2}+L_{3} \frac{\delta}{\gamma} e^{\gamma T} N_{2}\right) \mid y_{\infty}-y_{m}\right\|\right]\right.$

نعيد كتابة (40) ، (41) بشكل متجهات نجد ان

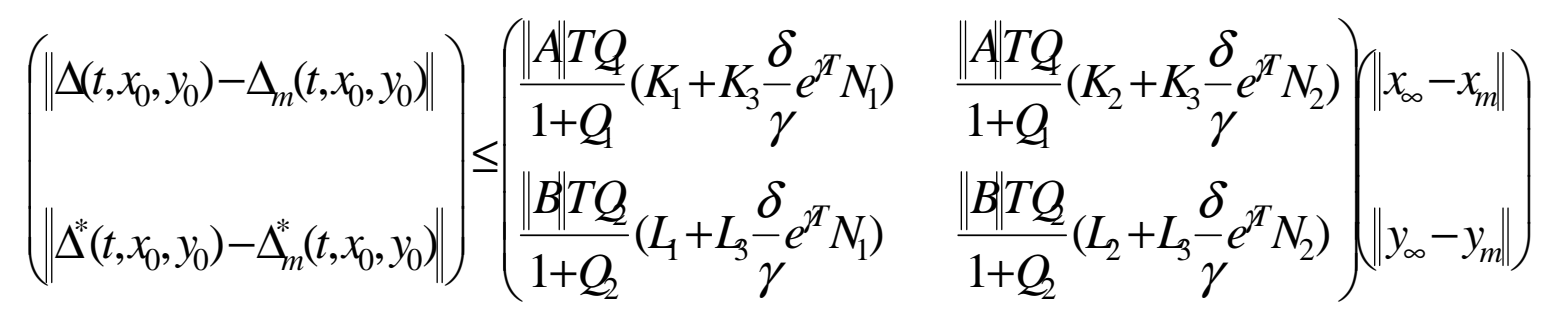

وباستخدام العلاقة (30) نحصل على (39) •

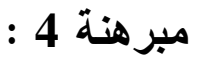

لنكن كل من الدالة (t,

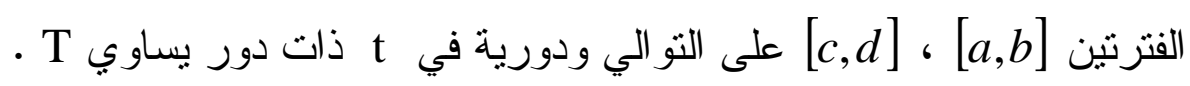
نفرض ان متتابعة الدو ال (37) تحقق المتباينتين الآتيتين: 


$$
\begin{aligned}
& \int_{\min }^{9} \text { رعد نوري بطرس وغادة شكر جميل } \\
& a+\frac{\|A\| T^{2} Q_{1}^{2}}{2\left(1+Q_{1}\right)} M_{1} \leq x_{0} \leq b-\frac{\|A\| T^{2} Q_{1}^{2}}{2\left(1+Q_{1}\right)} M_{1} \\
& \Delta_{m}\left(0, x_{0}, y_{0}\right) \leq-\delta \\
& \max _{\|A\| T^{2} Q_{1}^{2}} \quad \Delta_{m}\left(0, x_{0}, y_{0}\right) \geq \delta_{m} \\
& a+\frac{\|A\| T^{2} Q_{1}^{2}}{2\left(1+Q_{1}\right)} M_{1} \leq x_{0} \leq b-\frac{\|A\| T^{2} Q_{1}^{2}}{2\left(1+Q_{1}\right)} M_{1} \\
& \text { ونفرض ان متتابعة الدوال (38) تحقق المتباينتين الآتيتين: } \\
& \min _{\|B\| T^{2} Q_{2}^{2}} \min _{M_{2} \leq y_{0} \leq d-T^{2} Q_{2}^{2}} \Delta_{m}^{*}\left(0, x_{0}, y_{0}\right) \leq-\varepsilon \\
& c+\frac{\|B\| T^{2} Q_{2}^{2}}{2\left(1+Q_{2}\right)} M_{2} \leq y_{0} \leq d-\frac{\|B\| T^{2} Q_{2}^{2}}{2\left(1+Q_{2}\right)} M_{2} \\
& c+\frac{\|B\| T^{2} Q_{2}^{2}}{2\left(1+Q_{2}\right)} M_{2} \leq y_{0} \leq d-\frac{\|B\| T^{2} Q_{2}^{2}}{2\left(1+Q_{2}\right)} M_{2} \Delta_{m}^{*}\left(0, x_{0}, y_{0}\right) \geq \varepsilon_{m} \\
& \delta_{m}=\left[\frac{\|A\| T Q_{1}}{1+Q_{1}}\left(K_{1}+K_{3} \frac{\delta}{\gamma} e^{\gamma T} N_{1}\right)+\frac{\|A\| T Q_{1}}{1+Q_{1}}\left(K_{2}+K_{3} \frac{\delta}{\gamma} e^{\gamma T} N_{2}\right)\right] \Lambda^{m}(E-\Lambda)^{-1} \frac{\|A\| T^{2} Q_{1}^{2}}{2\left(1+Q_{1}\right)} M_{1} \\
& \varepsilon_{m}=\left[\frac{\|B\| T Q_{2}}{1+Q_{2}}\left(L_{1}+L_{3} \frac{\delta}{\gamma} e^{\gamma T} N_{1}\right)+\frac{\|B\| T Q_{2}}{1+Q_{2}}\left(L_{2}+L_{3} \frac{\delta}{\gamma} e^{\gamma T} N_{2}\right)\right] \Lambda^{m}(E-\Lambda)^{-1} \frac{\|B\| T^{2} Q_{2}^{2}}{2\left(1+Q_{2}\right)} M_{2} \\
& \text { عندئذ يكون للنظام (1) حل دوري لy } \\
& a+\frac{\|A\| T^{2} Q_{1}^{2}}{2\left(1+Q_{1}\right)} M_{1} \leq x(0) \leq b-\frac{\|A\| T^{2} Q_{1}^{2}}{2\left(1+Q_{1}\right)} M_{1}, c+\frac{\|B\| T^{2} Q_{2}^{2}}{2\left(1+Q_{2}\right)} M_{2} \leq y(0) \leq d-\frac{\|B\| T^{2} Q_{2}^{2}}{2\left(1+Q_{2}\right)} M_{2} \\
& \text { لتكن } \\
& \Delta_{m}\left(0, x_{1}, y_{1}\right)=\min _{a+\frac{\|A\| T^{2} Q_{1}^{2}}{2\left(1+Q_{1}\right)} M_{1} \leq x_{0} \leq b-\frac{\|A\| T^{2} Q_{1}^{2}}{2\left(1+Q_{1}\right)} M_{1}} \Delta_{m}\left(0, x_{0}, y_{0}\right) \\
& \left.\Delta_{m}\left(0, x_{2}, y_{2}\right)=\underset{a+\frac{\|A\| T^{2} Q_{1}^{2}}{2\left(1+Q_{1}\right)} M_{1} \leq x_{0} \leq b-\frac{\|A\| T^{2} Q_{1}^{2}}{2\left(1+Q_{1}\right)} M_{1}}{\max } \Delta_{m}\left(0, x_{0}, y_{0}\right)\right\}
\end{aligned}
$$

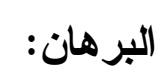

$$
\begin{aligned}
& \text { لتكن }
\end{aligned}
$$




$$
\begin{aligned}
& \text { [ل } \\
& \Delta_{m}^{*}\left(0, x_{1}, y_{1}\right)=\min _{c+\frac{\|B\| T^{2} Q_{2}^{2}}{2\left(1+Q_{2}\right)} M_{2} \leq y_{0} \leq d-\frac{\|B\| T^{2} Q_{2}^{2}}{2\left(1+Q_{2}\right)} M_{2}} \Delta_{m}^{*}\left(0, x_{0}, y_{0}\right) \\
& \left.\Delta_{m}^{*}\left(0, x_{2}, y_{2}\right)=\max _{c+\frac{\|B\| T^{2} Q_{2}^{2}}{2\left(1+Q_{2}\right)} M_{2} \leq y_{0} \leq d-\frac{\|B\| T^{2} Q_{2}^{2}}{2\left(1+Q_{2}\right)} M_{2}} \Delta_{m}^{*}\left(0, x_{0}, y_{0}\right)\right\}
\end{aligned}
$$

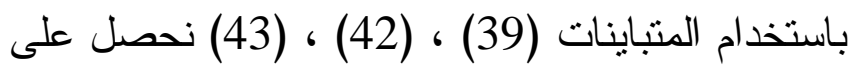

$$
\begin{aligned}
& \Delta\left(0, x_{1}, y_{1}\right)=\Delta_{m}\left(0, x_{1}, y_{1}\right)+\left[\Delta\left(0, x_{1}, y_{1}\right)-\Delta_{m}\left(0, x_{1}, y_{1}\right)\right]<0 \\
& \left.\Delta\left(0, x_{2}, y_{2}\right)=\Delta_{m}\left(0, x_{2}, y_{2}\right)+\left[\Delta\left(0, x_{2}, y_{2}\right)-\Delta_{m}\left(0, x_{2}, y_{2}\right)\right]>0\right\} \\
& \Delta^{*}\left(0, x_{1}, y_{1}\right)=\Delta_{m}^{*}\left(0, x_{1}, y_{1}\right)+\left[\Delta^{*}\left(0, x_{1}, y_{1}\right)-\Delta_{m}^{*}\left(0, x_{1}, y_{1}\right)\right]<0 \\
& \left.\Delta^{*}\left(0, x_{2}, y_{2}\right)=\Delta_{m}^{*}\left(0, x_{2}, y_{2}\right)+\left[\Delta^{*}\left(0, x_{2}, y_{2}\right)-\Delta_{m}^{*}\left(0, x_{2}, y_{2}\right)\right]>0\right\}
\end{aligned}
$$

ملاحظة 1 [ 5 ] :

تمت صياغة منطوق المبر هنة 4 مع البرهان عندما تكون $x_{0}$ كية غير متجهة. مبرهنة 5 :

لتكن كل من الدالتين $\Delta\left(0, x_{0}, y_{0}\right)=\int_{0}^{T} \frac{A}{E+e^{A T}} e^{A(T-s-\tau)} f\left(\tau, x_{\infty}\left(\tau, x_{0}, y_{0}\right), y_{\infty}\left(\tau, x_{0}, y_{0}\right), z_{\infty}\left(\tau, x_{0}, y_{0}\right)\right) d \tau$ $\Delta^{*}\left(0, x_{0}, y_{0}\right)=\int_{0}^{T} \frac{B}{E+e^{B T}} e^{B(T-s-\tau)} g\left(\tau, x_{\infty}\left(\tau, x_{0}, y_{0}\right), y_{\infty}\left(\tau, x_{0}, y_{0}\right), z_{\infty}\left(\tau, x_{0}, y_{0}\right)\right) d \tau$

إذ ان الدالتين (49) على التو الي، فإن المتباينات الآتية: لـ $\left\|\Delta\left(0, x_{0}, y_{0}\right)\right\| \leq M_{3}$ 


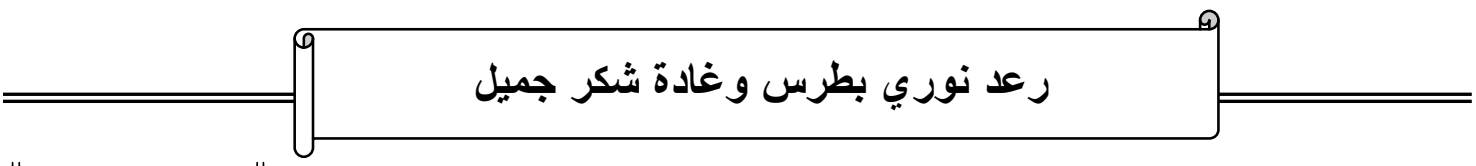

$$
\begin{aligned}
& \left\|\Delta^{*}\left(0, x_{0}, y_{0}\right)\right\| \leq M_{4} \\
& \left\|\Delta\left(0, x_{0}^{1}, y_{0}^{1}\right)-\Delta\left(0, x_{0}^{2}, y_{0}^{2}\right)\right\| \leq W_{6}\left\|x_{0}^{1}-x_{0}^{2}\right\|+W_{7}\left\|y_{0}^{1}-y_{0}^{2}\right\| \\
& \left\|\Delta^{*}\left(0, x_{0}^{1}, y_{0}^{1}\right)-\Delta^{*}\left(0, x_{0}^{2}, y_{0}^{2}\right)\right\| \leq W_{8}\left\|x_{0}^{1}-x_{0}^{2}\right\|+W_{9}\left\|y_{0}^{1}-y_{0}^{2}\right\| \\
& \text { - ت تحقق لكل } \\
& \text { حيث ان } \\
& W_{1}=\left(1-\frac{\| A \mid T^{2} Q_{1}^{2}}{2\left(1+Q_{1}\right)}\left(K_{1}+K_{3} \frac{\delta}{\gamma} e^{\mid T T} N_{1}\right)\right)^{-1} \quad, \quad W_{2}=\left(1-\frac{\|B\| T^{2} Q_{2}^{2}}{2\left(1+Q_{2}\right)}\left(L_{2}+L_{3} \frac{\delta}{\gamma} e^{2 T} N_{2}\right)\right)^{-1} \\
& W_{3}=\frac{\| A \mid T^{2} Q_{1}^{2}}{2\left(1+Q_{1}\right)}\left(K_{2}+K_{3} \frac{\delta}{\gamma} e^{2 T} N_{2}\right) W_{1} W_{2} \quad, \quad W_{4}=\left(1-\frac{\|B\| T^{2} Q_{2}^{2}}{2\left(1+Q_{2}\right)}\left(L_{1}+L_{3} \frac{\delta}{\gamma} e^{2 T} N_{1}\right)\right) W_{3} \\
& W_{5}=\frac{\|B\| T^{2} Q_{2}^{2}}{2\left(1+Q_{2}\right)}\left(L_{1}+L_{3} \frac{\delta}{\gamma} e^{\gamma T} N_{1}\right) \frac{W_{2}}{1-W_{4}} \\
& W_{6}=\left[\frac{\left(K_{1}+K_{3} \frac{\delta}{\gamma} e^{\gamma T} N_{1}\right)}{1-W_{4}}+\left(K_{2}+K_{3} \frac{\delta}{\gamma} e^{\gamma T} N_{2}\right) W_{5}\right] \frac{\|A\| T Q_{1}}{1+Q_{1}} W_{1} \\
& W_{7}=\left[\frac{\left(K_{1}+K_{3} \frac{\delta}{\gamma} e^{\gamma T} N_{1}\right)}{1-W_{4}}+\left(K_{2}+K_{3} \frac{\delta}{\gamma} e^{\gamma T} N_{2}\right)\left(1-W_{5}\right)\right] \frac{\|A\| T Q_{1}}{1+Q_{1}} W_{2} \\
& W_{8}=\left[\frac{\left(L_{1}+L_{3} \frac{\delta}{\gamma} e^{\gamma T} N_{1}\right)}{1-W_{4}}+\left(L_{2}+L_{3} \frac{\delta}{\gamma} e^{\gamma T} N_{2}\right) W_{5}\right] \frac{\| B \mid T Q_{2}}{1+Q_{2}} W_{1} \\
& W_{9}=\left[\frac{\left(L_{1}+L_{3} \frac{\delta}{\gamma} e^{\gamma T} N_{1}\right)}{1-W_{4}}+\left(L_{2}+L_{3} \frac{\delta}{\gamma} e^{\gamma T} N_{2}\right)\left(1-W_{5}\right)\right] \frac{\|B\| T Q_{2}}{1+Q_{2}} W_{2}
\end{aligned}
$$




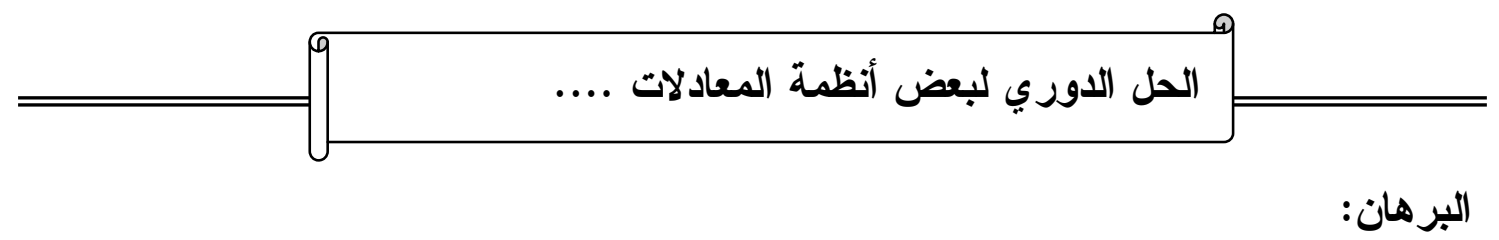

من صفات الدالتين كل من الدالتين

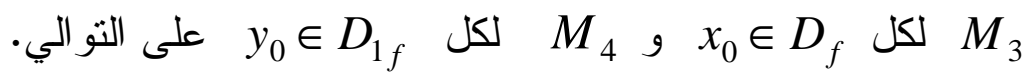
من العلاقة (48) فان المتباينة الآتية تتحقق

$$
\begin{aligned}
\left\|\Delta\left(0, x_{0}^{1}, y_{0}^{1}\right)-\Delta\left(0, x_{0}^{2}, y_{0}^{2}\right)\right\| \leq \frac{\|A\| T Q_{1}}{1+Q_{1}}\left[\left(K_{1}+K_{3} \frac{\delta}{\gamma} e^{\gamma T} N_{1}\right)\left\|x_{\infty}\left(t, x_{0}^{1}, y_{0}^{1}\right)-x_{\infty}\left(t, x_{0}^{2}, y_{0}^{2}\right)\right\|+\right. \\
\left.+\left(K_{2}+K_{3} \frac{\delta}{\gamma} e^{\gamma T} N_{2}\right)\left\|y_{\infty}\left(t, x_{0}^{1}, y_{0}^{1}\right)-y_{\infty}\left(t, x_{0}^{2}, y_{0}^{2}\right)\right\|\right]
\end{aligned}
$$

وكذلك بالطريقة نفسها، وبو اسطة (49) نحصل على:

$$
\begin{aligned}
\left\|\Delta^{*}\left(0, x_{0}^{1}, y_{0}^{1}\right)-\Delta^{*}\left(0, x_{0}^{2}, y_{0}^{2}\right)\right\| \leq \frac{\|B\| T Q_{2}}{1+Q_{2}} & {\left[\left(L_{1}+L_{3} \frac{\delta}{\gamma} e^{\gamma T} N_{1}\right) \mid x_{\infty}\left(t, x_{0}^{1}, y_{0}^{1}\right)-x_{\infty}\left(t, x_{0}^{2}, y_{0}^{2}\right) \|+\right.} \\
+ & \left.\left(L_{2}+L_{3} \frac{\delta}{\gamma} e^{\gamma T} N_{2}\right)\left\|y_{\infty}\left(t, x_{0}^{1}, y_{0}^{1}\right)-y_{\infty}\left(t, x_{0}^{2}, y_{0}^{2}\right)\right\|\right]
\end{aligned}
$$

بما ان

$$
\begin{aligned}
x\left(t, x_{0}^{\ell}, y_{0}^{\ell}\right)= & x_{0}^{\ell}+\int_{0}^{t} e^{A(t-s-\tau)}\left[f\left(\tau, x\left(\tau, x_{0}^{\ell}, y_{0}^{\ell}\right), y\left(\tau, x_{0}^{\ell}, y_{0}^{\ell}\right), z\left(\tau, x_{0}^{\ell}, y_{0}^{\ell}\right)\right)-\right. \\
& \left.-\int_{0}^{T} \frac{A}{E+e^{A T}} e^{A(T-s-\tau)} f\left(\tau, x\left(\tau, x_{0}^{\ell}, y_{0}^{\ell}\right), y\left(\tau, x_{0}^{\ell}, y_{0}^{\ell}\right), z\left(\tau, x_{0}^{\ell}, y_{0}^{\ell}\right)\right) d \tau\right] d \tau \\
y\left(t, x_{0}^{\ell}, y_{0}^{\ell}\right)= & y_{0}^{\ell}+\int_{0}^{t} e^{B(t-s-\tau)}\left[g\left(\tau, x\left(\tau, x_{0}^{\ell}, y_{0}^{\ell}\right), y\left(\tau, x_{0}^{\ell}, y_{0}^{\ell}\right), z\left(\tau, x_{0}^{\ell}, y_{0}^{\ell}\right)\right)-\right. \\
& \left.-\int_{0}^{T} \frac{B}{E+e^{B T}} e^{B(T-s-\tau)} g\left(\tau, x\left(\tau, x_{0}^{\ell}, y_{0}^{\ell}\right), y\left(\tau, x_{0}^{\ell}, y_{0}^{\ell}\right), z\left(\tau, x_{0}^{\ell}, y_{0}^{\ell}\right)\right) d \tau\right] d \tau
\end{aligned}
$$

$$
\text { حيث أن . }
$$

بما ان $\left\|x\left(t, x_{0}^{1}, y_{0}^{1}\right)-x\left(t, x_{0}^{2}, x_{0}^{2}\right)\right\| \leq W_{1}\left[\left\|x_{0}^{1}-x_{0}^{2}\right\|+\frac{\| A \mid T^{2} Q_{1}^{2}}{2\left(1+Q_{1}\right)}\left(K_{2}+K_{3} \frac{\delta}{\gamma} e^{\lambda T} N_{2}\right)\left\|y\left(t, x_{0}^{1}, y_{0}^{1}\right)-y\left(t, x_{0}^{2}, y_{0}^{2}\right)\right\|\right]$ 


$$
\begin{aligned}
& \text { لص رعد نوري بطرس وغادة شكر جميل } \\
& \text { بما ان } \\
& \left\|y\left(t, x_{0}^{1}, y_{0}^{1}\right)-y\left(t, x_{0}^{2}, x_{0}^{2}\right)\right\| \leq W_{2}\left[\left\|y_{0}^{1}-y_{0}^{2}\right\|+\frac{\|B\| T^{2} Q_{2}^{2}}{2\left(1+Q_{2}\right)}\left(L_{1}+L_{3} \frac{\delta}{\gamma} e^{\gamma T} N_{1}\right)\left\|x\left(t, x_{0}^{1}, y_{0}^{1}\right)-x\left(t, x_{0}^{2}, y_{0}^{2}\right)\right\|\right]
\end{aligned}
$$

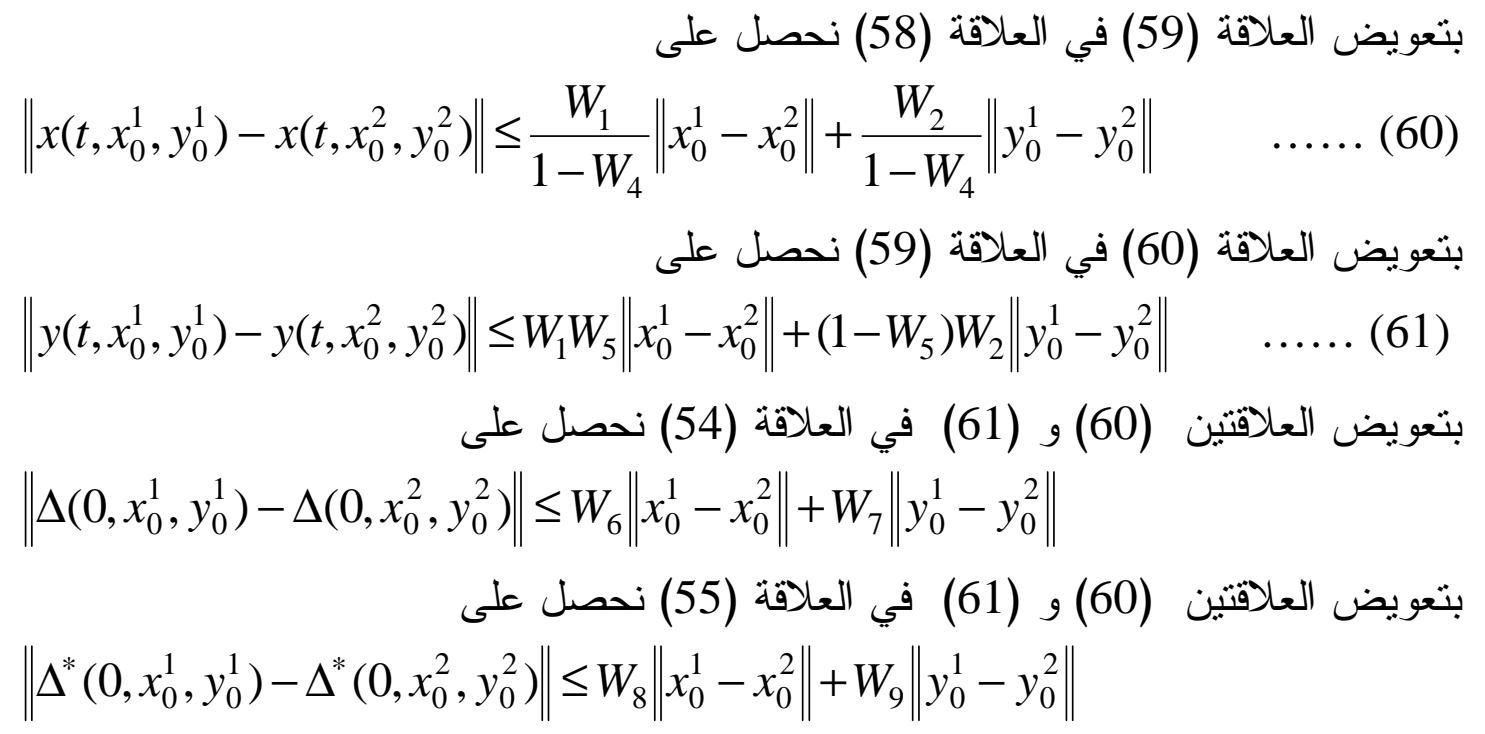

$$
\begin{aligned}
& \text { ملاحظة } 2 \text { [ [ ] }
\end{aligned}
$$

تؤكد المبرهنة 5 استقر ارية الحل للنظام (1)، وذللك عندما يحدث تغيير طفيف فـي$$
\text { النقطة } x_{0} \text { يقابله تغيير طفيف في سلوك الدالة }
$$

\section{REFERENCES}

1- Butris, R. N. and Ghada, Sh. J. The existence and approximation of periodic solution for systems of nonlinear integro-differential equations of voltarra type, Iraq, Mosul, J. of Educ. And sci, Vol.18, No.2 (2006), 104-114.

2- Butris, R. N. and Ghada, Sh. J. The existence and approximation of periodic solution for systems of nonlinear integro-differential equations of second order of voltarra type, Iraq, Mosul, J. of Educ. And sci, Vol.18, No.1 (2006), 124-137.

3- Kolmokorov, A. N. and Fomin, G. B. Introduction in the theory of functional and mathematical analysis, USSR, Moscow, (1989).

4- Martynyuk, D. I. Periodic solutions of second-order nonlinear differential equations, Ukraine, Kiev, Math. J. No. 4, (1967), 125-132.

5- Perestyuk, N. A. The Periodic solutions nonlinear systems of differential equations, Ukraine, Kiev, Math. And Meca. J., No. 5, (1971), 136-146.

6- Samoilenko, A. M. and Ronto, N. I. A numerical-analytic method for investigation of periodic solutions, Ukraine, Kiev, (1976). 